\title{
A covariance based framework for the propagation of uncertainty through inverse problems with an application to force identification
}

\author{
J.W.R. Meggitt ${ }^{1}$, A.T. Moorhouse ${ }^{1}$, A.S. Elliott ${ }^{1}$ \\ ${ }^{1}$ Acoustics Research Centre, University of Salford, Greater Manchester, M5 4WT
}

\begin{abstract}
Inverse problems are widely encountered in fields as diverse as physics, geophysics, engineering and finance. In the present paper, a covariance based framework for the estimation of their uncertainty is presented and applied to the problem of inverse force identification. A key step in its application involves the propagation of frequency response function (FRF) uncertainty through a matrix inversion, for example, between mobility and impedance. To this end a linearised inverse propagation relation is derived. This relation may be considered a generalisation of work presented in the particle physics literature, where we consider both complex valued and non-square matrices through a bivariate description of their uncertainty. Results are illustrated, first, through a numerical simulation where force and moment pairs are applied to a free-free beam model. An experimental study then illustrates the in-situ determination of blocked forces and their subsequent use in the prediction of an operational response. The uncertainties predicted by the proposed framework are in agreement with those acquired through Monte-Carlo (MC) methods for small input variance but are obtained at much lower computational cost, and with improved insight. In the process illustrating the propagation framework, matrix condition number, often taken as an indicator of uncertainty, is shown to relate poorly to a more rigorous uncertainty estimate, leaving open the question as to whether condition number is an appropriate indicator of uncertainty.
\end{abstract}

Keywords: Inverse methods, uncertainty propagation, in-situ blocked force, experimental structural dynamics

\section{Introduction}

The independent characterisation of vibratory sources (e.g. pumps, motors, etc.) has been of interest to those within the field of structural dynamics for many years [1]. Of the available quantities, there exist two fundamental descriptors of structural source activity, the blocked force and the free velocity [2]. The direct measurement of these quantities are, however, fraught with experimental difficulties and until recently neither had been considered a viable option for vibratory characterisation. In recent work by Moorhouse et al. [3] it was shown that the blocked force may be acquired not only indirectly, but in-situ, through an inverse procedure. The in-situ blocked force has since emerged as the most promising method towards the independent characterisation of structural sources. Its adoption within industry, and role in the formulation of the diagnostic procedure in-situ Transfer Path Analysis (TPA) [4], has led to numerous applications within the automotive $[5,6,7,8,9,10,11]$, aerospace, domestic product [12], and building acoustics [13] sectors. Whilst the in-situ blocked force approach has been well received, the uncertainties associated with its implementation have not yet been considered.

It is stated in the ISO supported document Guide [14] that, 'In general, the result of a measurement is only an approximation or estimate of the value of the measurand and thus is complete only when accompanied by a statement of the uncertainty of that estimate'. It is therefore the aim of this paper to establish a framework suitable for estimating the uncertainty associated with inversely determined blocked forces.

In contrast to many other fields, the estimation and propagation of uncertainty within experimental structural dynamics is complicated by: 1) the presence of complex variables, 2) their potentially correlated nature, and 3) the large number of degrees of freedom (DoFs) typically present. These factors must be acknowledged if an appropriate treatment of uncertainty is to be formulated. 
Whilst there exists a considerable body of literature concerning the quantification and propagation of uncertainty within numerical models (see $[15,16,17,18,19]$, to name but a few), the literature on experimental uncertainty appears comparatively sparse. The early work of Bendat et al. [20,21, 22] sees the formulation of the now common statistical error analysis relations used in the quantification of uncertainty in measured input/output relations, such as coherence and frequency response functions (FRFs). These relations, which are based on the assumption of small and uncorrelated uncertainty, have been used by many, particularly in the field of structural health monitoring [23, 24], to establish confidence bounds on measured FRFs that are only exceeded in the presence of damage. In more recent years the literature concerning FRF uncertainty has been driven by an interest in the fields of dynamic sub-structuring, modal analysis, and transfer path analysis. It has been shown that inconsistencies in measured FRF matrices can lead to: the extraction of incorrect modal parameters [25], spurious peaks in sub-structured FRF predictions [26, 27, 28], and large errors in reconstructed force estimates [29]. These examples further highlight the need to establish suitable methods for the treatment of uncertainty in experimental structural dynamics.

Interested in the effect of FRF uncertainty on dynamic sub-structuring procedures, de Klerk and Visser [30] attempted to characterise the experimental error associated with inconsistent excitation and response orientation, particularly in the measurement of driving point FRFs. The authors subsequently proposed an uncertainty propagation procedure for use in the prediction of coupled assembly FRFs [31,27]. Although the authors considered the separation of real and imaginary components using a complex matrix mapping, they chose not to account for their possible correlation, nor the correlation between the individual elements of the FRF matrix. Whilst in the presence of noise the entries of an FRF matrix may be assumed independent and identically distributed (i.i.d), and therefore uncorrelated, the uncertainty introduced as a result of human error (i.e. inconsistent force excitations) likely invalidates this assumption. In [32] Meggitt considered the effect of a random excitation position on the measurement of FRFs, in particular, on the correlation introduced between FRFs. Experimental evidence of this correlation, and its importance in the propagation of uncertainty, were demonstrated, the results of which suggest that a correct treatment of uncertainty should include the effects of inter-FRF correlation. To the author's knowledge no works have yet considered the correlated nature of FRF uncertainty on any eventual parameter estimation.

A related problem regarding the uncertainty of complex quantities was discussed in [33] where the authors were concerned with the estimation of complex reflection and transmission coefficients (i.e. s-parameters) encountered in radio frequency and microwave science. Their suggestion of using a bivariate description of complex uncertainty was further elaborated in [34]. In the field of structural dynamics, Schultz et al. [35] adopted the same complex bivariate description for FRFs acquired using a random noise excitation, and further investigated the propagation of uncertainty from real and imaginary components onto magnitude and phase descriptors. Similarly, Kim and Schmitz [36] used a complex bivariate description in the analysis of measured tool-holder-spindle-machine assembly FRFs.

Whilst several authors have investigated the nature of FRF uncertainty, few have considered its influence on the inverse determination of forces. Inverse methods, such as the in-situ blocked force approach considered in this work, are often assumed to be sensitive to error due to the effects of ill-conditioning. Many works can be found addressing this issue, particularly with regards to the suppression of its effects through methods such as regularisation $[37,38]$. In this field it appears to have become common practice to associate the condition number of measured FRF matrices with the degree of error, or uncertainty, as a result of ill-conditioning [39,40]. Whilst the condition number may indicate a rank deficiency, and thus the potential for ill-conditioning, in the presence of a realistic experimental uncertainty, it is not clear whether the condition number is an appropriate indicator of uncertainty. Nevertheless previous works have used the condition number as a means of propagating the uncertainty of measured FRFs and responses onto inversely determined force estimates [41]. With the condition number providing an upper bound on the propagation of relative error, these approaches typically overestimate the true level of uncertainty.

The inverse propagation of uncertainty was considered more generally by Fonseca et al. [42] where, as a means of avoiding the inversion of a propagation function, the authors posed the problem as a maximum likelihood optimisation. It was assumed that no correlation existed between the uncertain parameters and, furthermore, that an a priori estimate of the uncertain parameters was available. In [43], Zhang et al. tackled the problem of force reconstruction from a Bayesian perspective. Whilst the full posterior probability distribution of the reconstructed force is found, as per the Bayesian paradigm, some prior distribution must be elicited, which itself is not a trivial task. In [40] Wernsen et al. consider the effect of sensor noise, as opposed to FRF uncertainty, on the determination of blocked forces and discuss the importance of sensor positioning when preforming inverse force identification procedures.

In contrast to previous works, the proposed uncertainty framework is based on a complex bivariate law of error 
propagation. As such, it provides a more general, and arguably intuitive, inverse propagation of uncertainty, whilst correctly accounting for the complex and correlated nature of the associated parameters. Key in the formulation of the proposed framework is an expression derived for the propagation of uncertainty through a matrix inversion. The derivation and application of this relation to measured FRF matrices, in the context of blocked force uncertainty, may be considered one of the main contributions of this work.

The remainder of this paper will be structured as follows. Section 2 will begin by introducing and categorising the types of uncertainty encountered within experimental structural dynamics. Following this, section 3 will outline more specifically the blocked force inverse problem considered in this paper. In section 4 the laws of error propagation are formulated and applied to the problem at hand. Methods for the estimation of the required uncertainty parameters are discussed in section 5. In section 6 two demonstrative case studies are provided. Lastly, section 7 sees some concluding remarks.

\section{Categorisation of Uncertainty}

The uncertainties encountered within experimental structural dynamics may be categorised in a number of ways. Perhaps the most fundamental categorisation is between that of aleatory and epistemic. Aleatory uncertainties result from the inherent randomness of a system, for example the non-stationary operational behaviour of vibration sources. Aleatory uncertainty cannot be reduced and represents the intrinsic randomness of the phenomena under investigation. Epistemic uncertainties are those resulting from insufficient knowledge or information with regards to the modelling process, and include, for example, invalid assumptions in the equations of motion (i.e. linearity or time invariance). Epistemic uncertainty may in principle be reduced through the acquisition and implementation of additional information.

\begin{tabular}{lll}
\hline Type & Description & Category \\
\hline \hline 1) Model & $\begin{array}{l}\text { Uncertainty resulting from an approximate model being used to de- } \\
\text { scribe the physical problem. }\end{array}$ & Epistemic \\
2) Source & $\begin{array}{l}\text { Uncertainty inherent to the operational behaviour of the source. } \\
\text { 3) Experimental: }\end{array}$ & $\begin{array}{l}\text { Uncertainty due to experimentation. } \\
\text { 3a) Measurement }\end{array}$ \\
$\begin{array}{ll}\text { Due to the cumulative effect of noise sources in the measurement signal } \\
\text { path and computation post processing. }\end{array}$ & Aleatory \\
3b) Operator & Due to human error in the measurement procedure. & Epistemic
\end{tabular}

Table 1: Types of uncertainties encountered in experimental structural dynamic problems.

The categorisation of aleatory/epistemic, although general and well established in the literature [44], does not aid in identifying the source of uncertainty, nor its nature. In this work we will consider the type of uncertainty as being either model, source or experimentally based. This categorisations is summarised in table 1 and described below.

Model uncertainties arise when an approximate model is used to describe the physical problem, for example, assuming linearity and/or time invariance which is not realised in practice. Model uncertainty may be considered epistemic in that it stems from a lack of knowledge. Source uncertainty describes the inherent randomness of an operational source behaviour. Source uncertainty may be considered aleatory in that it is an intrinsic property of the source and cannot be reduced. Experimental uncertainty may be further subdivided into measurement and operator uncertainty. Measurement uncertainty describes the cumulative effect of noise sources within the measurement signal path, and beyond, for example, external disturbances, thermo-electrical noise, sampling error, finite precision, etc. Measurement uncertainty may be considered aleatory in that it is largely unavoidable but may be reduced to some extent. Operator uncertainty describes the effect of human error in the measurement procedure, for example, inconsistent location and/or direction of applied forces during the measurement of FRFs. Operator uncertainty may be considered epistemic as it may be reduced by carefully executed experimentation.

Regarding the nature of the above uncertainties, in the literature it is often assumed that experimental uncertainty is uncorrelated. Whilst this is likely a valid assumption in the case of measurement uncertainty, operator uncertainty has 
been shown to be correlated [32]. The nature of source uncertainty will vary depending upon the case considered, but will most likely be of a correlated nature. Similarly, model uncertainty will likely exhibit some form of correlation. Whilst the presence of correlated uncertainty complicates matters somewhat, its neglect has been shown to lead to large errors in uncertainty estimates [32].

\section{Inverse Problem}

Whilst the proposed uncertainty propagation framework is general to linear problems of the form $\mathbf{x}=\mathbf{A b}$, where $\mathbf{b}$ are inputs and $\mathbf{x}$ outputs, with $\mathbf{A}$ describing the influence of the system, we are concerned more specifically with the in-situ determination of blocked forces, and the uncertainties thereof. Our inverse problem therefore takes the form [3],

$$
\mathbf{v}_{\mathbf{b}}=\mathbf{Y}_{\mathbf{b c}} \overline{\mathbf{f}}_{\mathbf{c}}
$$

where $\mathbf{Y}_{\mathbf{b c}} \in \mathbb{C}^{N \times M}$ is the measured mobility matrix of a coupled assembly, $\mathbf{v}_{\mathbf{b}} \in \mathbb{C}^{N}$ is a measured operational velocity vector (note that accelerance and acceleration may be used in place of mobility and velocity), and $\overline{\mathbf{f}}_{\mathbf{c}} \in \mathbb{C}^{M}$ is the vector of unknown blocked forces. Here, subscripts $b$ and $c$ represent remote receiver and coupling interface DoFs, respectively (see figure 1).

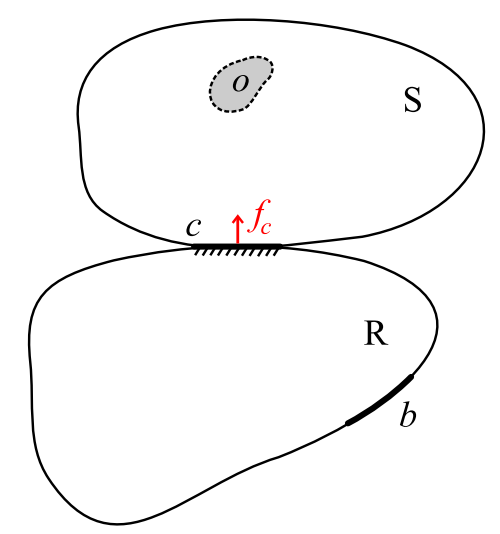

Figure 1: Diagram of a Source-Receiver (SR) structure illustrating the degrees of freedom (DoF) considered in equation 1. $c$ represents the set of interface DoFs, $b$ a set remote receiver DoFs, and $o$ the internal DoFs where the excitation mechanisms are present.

Variables are represented in the frequency domain, with the frequency variable omitted for clarity. For $N=M$, providing that the measured mobility matrix is of full rank, a unique solution is found through the inverse mobility matrix $\mathbf{Y}_{\mathbf{b c}}^{-\mathbf{1}}=\mathbf{Z}_{\mathbf{c b}}$, where $\mathbf{Z}_{\mathbf{c b}} \in \mathbb{C}^{N \times N}$ is an assembly impedance matrix. For $N>M$, the Moore-Penrose pseduo inverse [45] may be used in place of the classical matrix inverse to obtain $\mathbf{Y}_{\mathbf{b c}}^{+}=\mathbf{Z}_{\mathbf{c b}} \in \mathbb{C}^{M \times N}$, leading to a least squares solution of the problem. Noting the above, we are concerned more specifically with the propagation of uncertainty through the model,

$$
\overline{\mathbf{f}}_{\mathbf{c}}=\mathbf{Z}_{\mathbf{c b}} \mathbf{v}_{\mathbf{b}}
$$

The experimental implementation of equation 2 requires a two part measurement procedure. In the first, the source is turned on and the operational velocity, $\mathbf{v}_{\mathbf{b}}$, is measured. In the second, the source is turned off and the mobility matrix, $\mathbf{Y}_{\mathbf{b c}}$, is measured, from which the impedance, $\mathbf{Z}_{\mathbf{c b}}$, is calculated. A more detailed discussion regarding the implementation and experimental challenges associated with the in-situ determination of blocked forces may be found in [46].

At this point it is useful to identify the uncertainties associated with the model input parameters, $\mathbf{Z}_{\mathbf{c b}}$ and $\mathbf{v}_{\mathbf{b}}$. The notion of model uncertainty encompasses the whole of equation 2, in that the equation itself is an (approximate) model of the physical problem. Examples of model uncertainty include non-linearity and non-stationary source behaviour. 
however, the most likely/significant contributor is the incorrect representation of interface dynamics, e.g. the neglect of DoFs such as moments and/or in plane forces. Source uncertainty concerns the operational behaviour of the source and is therefore inherent only to the operational velocity $\mathbf{v}_{\mathbf{b}}$. Measurement uncertainty is associated with any measured quantity and therefore affects both the velocity and the impedance, $\mathbf{v}_{\mathbf{b}}$ and $\mathbf{Z}_{\mathbf{c b}}$, respectively. Operator uncertainty only affects quantities where continued human interaction is required and thus applies only to the impedance (or more directly, the mobility).

For generality, it is useful to consider the uncertainties associated with equation 2 as 'active' or 'passive'. Active uncertainties are those that describes the variability in the operational quantities (i.e. the velocity). Passive uncertainties describe the variability in the measured system properties (i.e. the impedance). The contribution of model, source and experimental uncertainty to active and passive quantities are summarised in table 2.

\begin{tabular}{ccccc}
\hline Quantity Type & Model & Source & \multicolumn{2}{c}{ Experimental } \\
& & & Measurement & Operator \\
\hline \hline Active & $x$ & $\checkmark$ & $\checkmark$ & $x$ \\
Passive & $x$ & $x$ & $\checkmark$ & $\checkmark$ \\
\hline
\end{tabular}

Table 2: Contribution of model, source and experimental uncertainty to active and passive quantities. Note that model uncertainty encompass the whole of equation 2 and is therefore neither active nor passive.

\subsection{A Note on Model Uncertainty}

Whilst neither the quantification nor the propagation of model uncertainty will be considered in detail in this paper, a brief discussion with regards to its most likely origin is warranted. Here, this origin is considered a consequence of an incomplete interface description (i.e the neglect of a subset of coupling interface DoFs), and will be demonstrated below. The following derivation is summarised from [47] for completeness.

Consider equation 1 in the form,

$$
\mathbf{v}_{\mathbf{b}}=\mathbf{Y}_{\mathbf{b c}} \overline{\mathbf{f}}_{\mathbf{c}}+\mathbf{Y}_{\mathbf{b} \hat{\mathbf{c}}} \overline{\mathbf{f}}_{\hat{\mathbf{c}}}
$$

where the velocity contributions from known and unknown DoFs have been partitioned. Here, $c$ represents the coupling interface DoFs that are known and considered measurable, whilst $\hat{c}$ represents the interface DoFs that are unknown, or known but can not be measured (for example, rotational and/or in-plane).

In the experimental determination of the blocked force one pre-multiplies the operational velocity vector by the inverse mobility matrix pertaining to the known DoFs. Pre-multiplication of equation 3 by $\mathbf{Y}_{\mathbf{b c}}^{-\mathbf{1}}$ thus yields,

$$
\mathbf{Y}_{\mathbf{b c}}^{-1} \mathbf{v}_{\mathbf{b}}=\overline{\mathbf{f}}_{\mathbf{c}}+\mathbf{Y}_{\mathbf{b c}}^{-1} \mathbf{Y}_{\mathbf{b c}} \overline{\mathbf{f}}_{\hat{\mathbf{c}}}=\tilde{\overline{\mathbf{f}}}_{\mathbf{c}}
$$

where $\tilde{\overline{\mathbf{f}}}_{\mathbf{c}}$ represents the acquired blocked force. Although correct in its own respect, $\tilde{\overline{\mathbf{f}}}_{\mathbf{c}}$ is not the true blocked force. ${ }^{1}$ The neglect of the DoFs $\hat{c}$ has resulted in the additional term, $\mathbf{Y}_{\mathbf{b c}}^{-\mathbf{1}} \mathbf{Y}_{\mathbf{b} \hat{\mathbf{c}}} \overline{\mathbf{f}}_{\hat{\mathbf{c}}}$. This term is a property of the coupled assembly. Consequently, the acquired blocked force is no longer an independent property of the source. This loss of independence may have severe implications when the acquired blocked force is used to predict the operational response in a new assembly. This is demonstrated below.

Suppose we have acquired the set of blocked forces, $\tilde{\mathbf{f}}_{\mathbf{c}}$, from a test rig through the above procedure. We are interested in predicting the operational response in a new installation, i.e. the remote receiver velocity, $\mathbf{v}_{\mathbf{b}}^{\prime}$ (where the prime symbol, ', is used to denote the assembly of the new installation). The true velocity, that we are aiming to predict, may be expressed in terms of the true blocked force as,

$$
\mathbf{v}_{\mathbf{b}}^{\prime}=\mathbf{Y}_{\mathbf{b c}}^{\prime} \overline{\mathbf{f}}_{\mathbf{c}}+\mathbf{Y}_{\mathbf{b} \hat{\mathbf{c}}}^{\prime} \overline{\mathbf{f}}_{\hat{\mathbf{c}}}
$$

\footnotetext{
${ }^{1}$ The blocked force $\tilde{\mathbf{f}}_{\text {Sc }}$ represents the reaction forces due to a source that is blocked in the $c$ DoFs, but unrestrained in the remaining $\hat{c}$ DoFs. It is therefore by definition a blocked force, albeit not the true blocked force.
} 
To predict this remote velocity we pre-multiply our acquired blocked force, $\tilde{\overline{\mathbf{f}}}_{\mathbf{c}}$, by the coupled transfer mobility that corresponds to the known DoFs, $c$, on the new installation, $\mathbf{Y}_{\mathbf{b c}}^{\prime}$.

$$
\tilde{\mathbf{v}}_{\mathbf{b}}^{\prime}=\mathbf{Y}_{\mathbf{b c}}^{\prime} \tilde{\overline{\mathbf{f}}}_{\mathbf{c}}=\mathbf{Y}_{\mathbf{b c}}^{\prime}\left(\overline{\mathbf{f}}_{\mathbf{c}}+\mathbf{Y}_{\mathbf{b c}}^{-1} \mathbf{Y}_{\mathbf{b} \hat{\mathbf{c}}} \overline{\mathbf{f}}_{\hat{\mathbf{c}}}\right)
$$

Expanding the above we arrive at,

$$
\tilde{\mathbf{v}}_{\mathbf{b}}^{\prime}=\mathbf{Y}_{\mathbf{b c}}^{\prime} \overline{\mathbf{f}}_{\mathbf{c}}^{\prime}+\mathbf{Y}_{\mathbf{b c}}^{\prime} \mathbf{Y}_{\mathbf{b c}}^{-1} \mathbf{Y}_{\mathbf{b} \mathbf{c}} \overline{\mathbf{f}}_{\hat{\mathbf{c}}}
$$

From equation 5 and 7 it is clear that the true velocity, $\mathbf{v}_{\mathbf{b}}^{\prime}$, and the predicted velocity, $\tilde{\mathbf{v}}_{\mathbf{b}}^{\prime}$, are not equal. Hence the blocked force is no longer transferable and the predicted velocity is subject to a degree of model uncertainty.

The above analysis clearly illustrates that model uncertainty is introduced through the neglect of coupling DoFs, i.e. an incomplete interface description. The consequence of an incomplete interface description will vary depending upon the influence of the neglected DoFs. Nevertheless, efforts should be made to minimise its impact. With this in mind, an experimental test for completeness was recently proposed by Meggitt et al. [47], where the authors used an Interface Completeness Criterion to assess the importance of neglected interface DoFs, and quantify the completeness of a given interface description.

Further investigation into the effect of model uncertainty is considered beyond the scope of this work and, as such, the remainder of this paper will assume a complete interface description, thus eliminating this source of model uncertainty. We will therefore consider the propagation of uncertainty in terms of the active and passive uncertainties only.

\section{Propagation of Active and Passive Uncertainty}

In this section we will derive the appropriate formulae for propagating active and passive uncertainty through the inverse problem, as represented by equation 2. Hereafter, for clarity, subscripts $b$ and $c$ (which represent sets of DoFs) will be dropped, instead subscripts $i$ and $j$ will be used to denote the $i$ th and $j$ th elements of the marked vector (or matrix).

The law of error propagation is given in its general form by,

$$
\sigma_{x_{i} x_{j}}=\mathbf{J} \boldsymbol{\Sigma}_{\mathbf{y}} \mathbf{J}^{\mathbf{T}}
$$

where; $\sigma_{x_{i} x_{j}}$ is the covariance between elements $i$ and $j$ of the output variable $\mathbf{x}, \boldsymbol{\Sigma}_{\mathbf{y}}$ is the variance-covariance matrix ${ }^{2}$ of the input variable $\mathbf{y}$, and $\mathbf{J}$ is the Jacobian associated with the propagation model (i.e. equation 2). For completeness the above expression is derived in Appendix A. When applying the law of error propagation it is important to note that it is based on the assumption of small variance, i.e. it is formulated from a first order approximation of the propagating function.

In what follows the law of error propagation will be applied to the inverse problem of equation 2 . In order to correctly account for the complex nature of the frequency domain representation, a bivariate description of complex uncertainty will be adopted [34, 32].

An element $i$ of the blocked force vector $\overline{\mathbf{f}} \in \mathbb{C}^{M}$ may be considered, generally, as the output of a complex function, $G()$, of input variables, $\mathbf{P}_{\mathbf{i}} \in \mathbb{C}^{2 N}$.

$$
\bar{f}_{i}=G\left(Z_{i 1}, Z_{i 2}, \cdots, Z_{i N} \mid v_{1}, v_{2}, \cdots, v_{N}\right)=G\left(\mathbf{P}_{\mathbf{i}}\right)=\sum_{n=1}^{N} Z_{i n} v_{n}
$$

Alternatively, the complex function, $G()$, may be expressed as a pair of scalar functions that separately evaluate the real and imaginary components of the blocked force (i.e. $\left.\bar{f}_{i}=G_{\mathfrak{R}}\left(\mathbf{P}_{\mathbf{i}}\right)+i G_{\mathfrak{I}}\left(\mathbf{P}_{\mathbf{i}}\right)\right)$. Consequently, the real and imaginary components of the blocked force element $i$ are given by,

$$
\mathfrak{R}\left(\bar{f}_{i}\right)=G_{\mathfrak{R}}\left(\mathbf{P}_{\mathbf{i}}\right)=G_{\mathfrak{R}}\left(\mathfrak{R}\left(Z_{i 1}\right), \mathfrak{J}\left(Z_{i 1}\right), \cdots, \mathfrak{R}\left(Z_{i N}\right), \mathfrak{J}\left(Z_{i N}\right) \mid \mathfrak{R}\left(v_{1}\right), \mathfrak{J}\left(v_{1}\right), \cdots \mathfrak{R}\left(v_{N}\right), \mathfrak{J}\left(v_{N}\right)\right)
$$

\footnotetext{
${ }^{2}$ Hereafter we will use the term covariance matrix generally to describe both covariance and variance-covariance matrices.
} 
and

$$
\mathfrak{J}\left(\bar{f}_{i}\right)=G_{\mathfrak{I}}\left(\mathbf{P}_{\mathbf{i}}\right)=G_{\mathfrak{I}}\left(\mathfrak{R}\left(Z_{i 1}\right), \mathfrak{J}\left(Z_{i 1}\right), \cdots, \mathfrak{R}\left(Z_{i N}\right), \mathfrak{J}\left(Z_{i N}\right) \mid \mathfrak{R}\left(v_{1}\right), \mathfrak{J}\left(v_{1}\right), \cdots \mathfrak{R}\left(v_{N}\right), \mathfrak{J}\left(v_{N}\right)\right)
$$

where the input variable $\mathbf{P}_{\mathbf{i}}$ is separated into its real and imaginary components. The Jacobians associated with the above scalar functions are given, respectively, by,

$$
\mathbf{J}_{\mathfrak{R}_{\mathbf{i}}}=\left[\begin{array}{llllllllll}
\frac{\partial G_{\mathfrak{R}}\left(\mathbf{P}_{\mathbf{i}}\right)}{\partial \mathfrak{R}\left(Z_{i 1}\right)} & \frac{\partial G_{\mathfrak{R}}\left(\mathbf{P}_{\mathbf{i}}\right)}{\partial \mathfrak{I}\left(Z_{i 1}\right)} & \cdots & \frac{\partial G_{\mathfrak{R}}\left(\mathbf{P}_{\mathbf{i}}\right)}{\partial \mathfrak{R}\left(Z_{i N}\right)} & \frac{\partial G_{\mathfrak{R}}\left(\mathbf{P}_{\mathbf{i}}\right)}{\partial \mathfrak{I}\left(Z_{i N}\right)} & \frac{\partial G_{\mathfrak{R}}\left(\mathbf{P}_{\mathbf{i}}\right)}{\partial \mathfrak{R}\left(v_{1}\right)} & \frac{\partial G_{\mathfrak{R}}\left(\mathbf{P}_{\mathbf{i}}\right)}{\partial \mathfrak{I}\left(v_{1}\right)} & \cdots & \frac{\partial G_{\mathfrak{R}}\left(\mathbf{P}_{\mathbf{i}}\right)}{\partial \mathfrak{R}\left(v_{N}\right)} & \frac{\partial G_{\mathfrak{R}}\left(\mathbf{P}_{\mathbf{i}}\right)}{\partial \mathfrak{I}\left(v_{N}\right)}
\end{array}\right]
$$

and

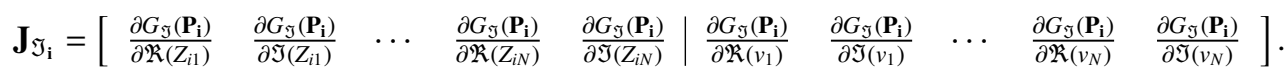

In the presence of complex variables the propagation model of interest (i.e. equation 2) may be expressed using a complex matrix mapping [34] in the form,

$$
\left(\begin{array}{c}
\mathfrak{R}\left(\bar{f}_{i}\right) \\
\mathfrak{J}\left(\bar{f}_{i}\right)
\end{array}\right)=\left[\begin{array}{ccccc}
\mathfrak{R}\left(Z_{i 1}\right) & -\mathfrak{J}\left(Z_{i 1}\right) & \ldots & \mathfrak{R}\left(Z_{i N}\right) & -\mathfrak{J}\left(Z_{i N}\right) \\
\mathfrak{J}\left(Z_{i 1}\right) & \mathfrak{R}\left(Z_{i 1}\right) & \ldots & \mathfrak{I}\left(Z_{i N}\right) & \mathfrak{R}\left(Z_{i N}\right)
\end{array}\right]\left(\begin{array}{c}
\mathfrak{R}\left(v_{1}\right) \\
\mathfrak{I}\left(v_{1}\right) \\
\vdots \\
\mathfrak{R}\left(v_{N}\right) \\
\mathfrak{J}\left(v_{N}\right)
\end{array}\right) .
$$

Noting that equation 14 represents a linear combination of impedance and velocity terms, its clear that its derivative with respect to velocity will yield an impedance, and vice versa. As such, equations 12 and 13 may be evaluated and expressed in a similar matrix form,

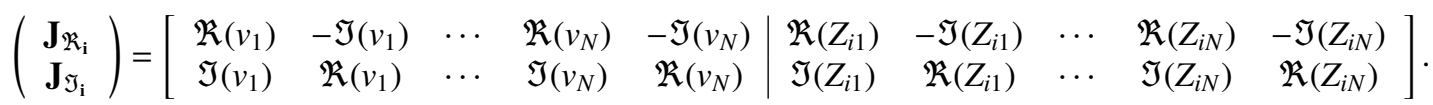

The above procedure is repeated for a second DoF, $j$, such that the blocked force, $\bar{f}_{j}$, and its associated Jacobian are given, respectively, by,

$$
\left(\begin{array}{c}
\mathfrak{R}\left(\bar{f}_{j}\right) \\
\mathfrak{I}\left(\bar{f}_{j}\right)
\end{array}\right)=\left[\begin{array}{ccccc}
\mathfrak{R}\left(Z_{j 1}\right) & -\mathfrak{J}\left(Z_{j 1}\right) & \ldots & \mathfrak{R}\left(Z_{j N}\right) & -\mathfrak{I}\left(Z_{j N}\right) \\
\mathfrak{J}\left(Z_{j 1}\right) & \mathfrak{R}\left(Z_{j 1}\right) & \ldots & \mathfrak{J}\left(Z_{j N}\right) & \mathfrak{R}\left(Z_{j N}\right)
\end{array}\right]\left(\begin{array}{c}
\mathfrak{R}\left(v_{1}\right) \\
\mathfrak{I}\left(v_{1}\right) \\
\vdots \\
\mathfrak{R}\left(v_{N}\right) \\
\mathfrak{J}\left(v_{N}\right)
\end{array}\right)
$$

and,

$$
\left(\begin{array}{c}
\mathbf{J}_{\mathfrak{R}_{\mathbf{j}}} \\
\mathbf{J}_{\mathfrak{J}_{\mathfrak{j}}}
\end{array}\right)=\left[\begin{array}{ccccc|ccccc}
\mathfrak{R}\left(v_{1}\right) & -\mathfrak{J}\left(v_{1}\right) & \ldots & \mathfrak{R}\left(v_{N}\right) & -\mathfrak{J}\left(v_{N}\right) & \mathfrak{R}\left(Z_{j 1}\right) & -\mathfrak{I}\left(Z_{j 1}\right) & \ldots & \mathfrak{R}\left(Z_{j N}\right) & -\mathfrak{J}\left(Z_{j N}\right) \\
\mathfrak{J}\left(v_{1}\right) & \mathfrak{R}\left(v_{1}\right) & \ldots & \mathfrak{J}\left(v_{N}\right) & \mathfrak{R}\left(v_{N}\right) & \mathfrak{J}\left(Z_{j 1}\right) & \mathfrak{R}\left(Z_{j 1}\right) & \ldots & \mathfrak{J}\left(Z_{j N}\right) & \mathfrak{R}\left(Z_{j N}\right)
\end{array}\right] .
$$

As per the law of error propagation, the complex bivariate covariance matrix between the blocked force elements $i$ and $j$ is given by,

$$
\boldsymbol{\Sigma}_{\overline{\mathbf{f}}_{\mathbf{i}} \overline{\mathbf{f}}_{\mathbf{j}}}=\left[\begin{array}{cc}
\sigma_{\mathfrak{R}\left(\bar{f}_{i}\right) \mathfrak{R}\left(\bar{f}_{j}\right)} & \sigma_{\mathfrak{R}\left(\bar{f}_{i}\right) \mathfrak{I}\left(\bar{f}_{j}\right)} \\
\sigma_{\mathfrak{J}\left(\bar{f}_{i}\right) \mathfrak{R}\left(\bar{f}_{j}\right)} & \sigma_{\mathfrak{I}\left(\bar{f}_{i}\right) \mathfrak{I}\left(\bar{f}_{j}\right)}
\end{array}\right]=\left(\begin{array}{c}
\mathbf{J}_{\mathfrak{R}_{\mathbf{i}}} \\
\mathbf{J}_{\mathfrak{J}_{\mathbf{i}}}
\end{array}\right) \boldsymbol{\Sigma}_{\mathbf{P}_{\mathbf{i}} \mathbf{P}_{\mathbf{j}}}\left(\begin{array}{ll}
\mathbf{J}_{\mathfrak{R}_{\mathbf{j}}}^{\mathbf{T}} & \mathbf{J}_{\mathfrak{J}_{\mathbf{j}}}^{\mathbf{T}}
\end{array}\right)
$$


where the input variable covariance matrix, $\boldsymbol{\Sigma}_{\mathbf{P}_{\mathbf{i}} \mathbf{P}_{\mathbf{j}}} \in \mathbb{R}^{4 N \times 4 N}$, is given by

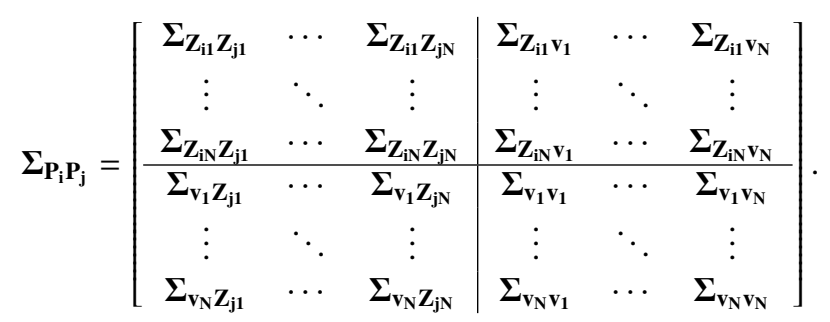

Note that each entry of the covariance matrix $\boldsymbol{\Sigma}_{\mathbf{P}_{\mathbf{i}} \mathbf{P}_{\mathbf{j}}}$ represents a complex bivariate covariance matrix between the subscripted variables,

$$
\boldsymbol{\Sigma}_{\mathbf{x y}}=\left[\begin{array}{cc}
\sigma_{\mathfrak{R}(x) \mathfrak{R}(y)} & \sigma_{\mathfrak{R}(x) \mathfrak{I}(y)} \\
\sigma_{\mathfrak{I}(x) \mathfrak{R}(y)} & \sigma_{\mathfrak{J}(x) \mathfrak{I}(y)}
\end{array}\right] .
$$

We will continue under the assumption that the operational velocity and impedance are uncorrelated. This is a reasonable assumption since the velocity is dependent on the operational activity of the source, whilst the impedance is not. Note however that we retain the possibility of correlation within both the operational velocity vector and the impedance matrix.

A complex bivariate uncertainty propagation is consequently given by,

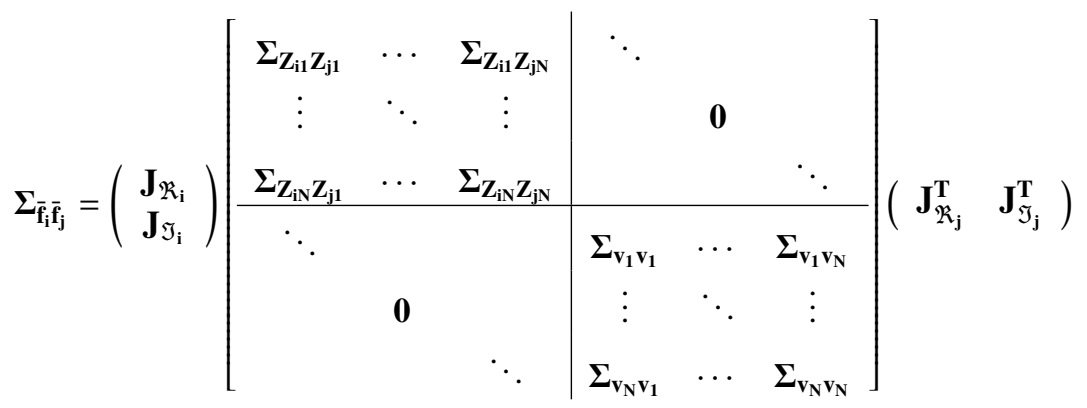

or more compactly as,

$$
\boldsymbol{\Sigma}_{\overline{\mathbf{f}}_{\mathrm{i}} \overline{\mathbf{f}}_{\mathbf{j}}}=\left(\begin{array}{c|c}
\mathbf{J}_{\mathfrak{R}_{\mathrm{i}}} \\
\mathbf{J}_{\mathfrak{J}_{\mathrm{i}}}
\end{array}\right)\left[\begin{array}{c|c}
\boldsymbol{\Sigma}_{\mathbf{Z}_{\mathrm{i}} \mathbf{Z}_{\mathrm{j}}:} & \mathbf{0} \\
\hline \mathbf{0} & \boldsymbol{\Sigma}_{\mathbf{v}}
\end{array}\right]\left(\begin{array}{cc}
\mathbf{J}_{\mathfrak{R}_{\mathrm{j}}}^{\mathbf{T}} & \mathbf{J}_{\mathfrak{J}_{\mathrm{j}}}^{\mathbf{T}}
\end{array}\right)
$$

where the colon subscript : is used to denote an entire column of the respective matrix.

Noting that the partitioned covariance matrix $\boldsymbol{\Sigma}_{\mathbf{v}} \in \mathbb{R}^{2 N \times 2 N}$ is independent of the indices $i$ and $j$ (i.e. the same velocities are used to calculate each blocked force), equation 22 may be extended such that the entire blocked force covariance matrix is given by,

$$
\left[\begin{array}{ccc}
\boldsymbol{\Sigma}_{\overline{\mathbf{f}}_{1} \overline{\mathbf{f}}_{1}} & \cdots & \boldsymbol{\Sigma}_{\overline{\mathbf{f}}_{1} \overline{\mathbf{f}}_{\mathrm{M}}} \\
\vdots & \ddots & \vdots \\
\boldsymbol{\Sigma}_{\overline{\mathbf{f}}_{\mathbf{M}} \overline{\mathbf{f}}_{1}} & \cdots & \boldsymbol{\Sigma}_{\overline{\mathbf{f}}_{\mathbf{M}} \overline{\mathbf{f}}_{\mathbf{M}}}
\end{array}\right]=\mathbf{J}\left[\begin{array}{ccc|c}
\boldsymbol{\Sigma}_{\mathbf{Z}_{1: \mathbf{Z}_{1}:}} & \cdots & \boldsymbol{\Sigma}_{\mathbf{Z}_{1:} \mathbf{Z}_{\mathbf{M}}:} & \mathbf{0} \\
\vdots & \ddots & \vdots & \vdots \\
\boldsymbol{\Sigma}_{\mathbf{Z}_{\mathrm{M}} \mathbf{Z}_{1:}} & \cdots & \boldsymbol{\Sigma}_{\mathbf{Z}_{\mathrm{M}: \mathbf{Z}_{M}:}} & \mathbf{0} \\
\hline \mathbf{0} & \cdots & \mathbf{0} & \boldsymbol{\Sigma}_{\mathbf{v}}
\end{array}\right] \mathbf{J}^{\mathbf{T}}
$$

or more compactly as,

$$
\Sigma_{\overline{\mathbf{f}}}=\mathbf{J}\left[\begin{array}{c|c}
\Sigma_{\mathbf{Z}} & \mathbf{0} \\
\hline \mathbf{0} & \boldsymbol{\Sigma}_{\mathbf{v}}
\end{array}\right] \mathbf{J}^{\mathbf{T}}
$$

where, $\boldsymbol{\Sigma}_{\overline{\mathbf{f}}} \in \mathbb{R}^{2 M \times 2 M}$ is the complex bivariate covariance matrix of the blocked force vector $\overline{\mathbf{f}}, \boldsymbol{\Sigma}_{\mathbf{Z}} \in \mathbb{R}^{2 N M \times 2 N M}$ is the complex bivariate covariance matrix of the impedance matrix $\mathbf{Z}, \boldsymbol{\Sigma}_{\mathbf{v}} \in \mathbb{R}^{2 N \times 2 N}$ is the complex bivariate covariance 
matrix of the velocity vector $\mathbf{v}$, and $\mathbf{J} \in \mathbb{R}^{2 M \times(2 N M+2 N)}$ is the associated Jacobian matrix, given by,

$$
\mathbf{J}=\left[\begin{array}{ccc|c}
\mathbf{v} & & & \mathbf{Z}_{1:} \\
& \ddots & & \vdots \\
& & \mathbf{v} & \mathbf{Z}_{\mathbf{M}:}
\end{array}\right]
$$

where the velocity, $\mathbf{v} \in \mathbb{R}^{2 \times 2 N}$, and impedance, $\mathbf{Z}_{\mathbf{M}:} \in \mathbb{R}^{2 \times 2 N}$, are given in complex matrix form by

$$
\mathbf{v}=\left[\begin{array}{ccccc}
\mathfrak{R}\left(v_{1}\right) & -\mathfrak{J}\left(v_{1}\right) & \cdots & \mathfrak{R}\left(v_{N}\right) & -\mathfrak{I}\left(v_{N}\right) \\
\mathfrak{I}\left(v_{1}\right) & \mathfrak{R}\left(v_{1}\right) & \cdots & \mathfrak{I}\left(v_{N}\right) & \mathfrak{R}\left(v_{N}\right)
\end{array}\right]
$$

and

$$
\mathbf{Z}_{\mathbf{M}:}=\left[\begin{array}{ccccc}
\mathfrak{R}\left(Z_{M 1}\right) & -\mathfrak{J}\left(Z_{M 1}\right) & \cdots & \mathfrak{R}\left(Z_{M N}\right) & -\mathfrak{I}\left(Z_{M N}\right) \\
\mathfrak{J}\left(Z_{M 1}\right) & \mathfrak{R}\left(Z_{M 1}\right) & \cdots & \mathfrak{J}\left(Z_{M N}\right) & \mathfrak{R}\left(Z_{M N}\right)
\end{array}\right]
$$

respectively.

Equations 23-27 propagate the complex uncertainties associated with impedance, $\mathbf{Z}$, and velocity, $\mathbf{v}$, (in terms of their bivariate complex covariance matrices) through equation 2 , and onto the real and imaginary components of the blocked force, $\overline{\mathbf{f}}$. Equations 23-27 thus form the basis of the proposed inverse uncertainty propagation framework. It is worth noting that if the system considered is linear, i.e. $\overline{\mathbf{f}}_{\mathbf{c}}=\mathbf{Z}_{\mathbf{c b}} \mathbf{v}_{\mathbf{b}}$, the proposed framework provides an exact propagation of uncertainty, given that $\boldsymbol{\Sigma}_{\mathbf{Z}}$ and $\boldsymbol{\Sigma}_{\mathrm{v}}$ are themselves correct, since the propagation function, $\overline{\mathbf{f}}_{\mathbf{c}}=\mathbf{Z}_{\mathrm{cb}} \mathbf{v}_{\mathbf{b}}$, has only a first order derivative. That said, an approximation will be introduced as $\boldsymbol{\Sigma}_{\mathbf{Z}}$ is not directly available and must be obtained from $\boldsymbol{\Sigma}_{\mathbf{Y}}$ in some way, as will be discussed in the following section.

\subsection{Presentation of Complex Uncertainty}

When dealing with complex quantities, it is typical to present parameter estimates not in the form of their real and imaginary components, but in magnitude. As such, it would be beneficial to provide a measure of the total variability of each blocked force, as opposed to its real and imaginary components. Two common measures are the total dispersion (sum of variances) and the generalised variance (determinant of covariance matrix) [48]. Alternatively, the law of error propagation may be used to propagate the uncertainty of real and imaginary components onto the magnitude $\left(\left|\bar{f}_{i}\right|=\sqrt{\mathfrak{R}\left(\bar{f}_{i}\right)^{2}+\mathfrak{J}\left(\bar{f}_{i}\right)^{2}}\right)$

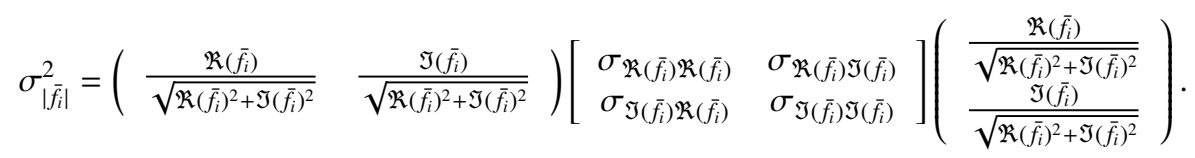

A similar procedure may be used to determine the uncertainty in phase angle, $\angle \bar{f}_{i}=\tan ^{-1}\left(\frac{\mathfrak{S}\left(\bar{f}_{i}\right)}{\mathcal{R}\left(\bar{f}_{i}\right)}\right)$, although care must be taken so as to ensure the correct quadrant of the complex plane is considered. A more thorough discussion regarding propagation of complex uncertainty onto magnitude and phase descriptors may be found in [35].

In summary, the above framework provides a general treatment of the uncertainties associated with inversely determined blocked forces. The presence of both complex and correlated uncertainties have been acknowledged and, moreover, the proposed framework may readily be extended to suit an arbitrary number of DoFs. The proposed framework will be used to estimate the uncertainties present in inversely determined forces as part of two case studies presented in section 6 . Firstly, however, the estimation of the required input uncertainties, namely, $\boldsymbol{\Sigma}_{\mathbf{v}}$ and $\boldsymbol{\Sigma}_{\mathbf{Z}}$, must be considered.

\section{Estimation of Active and Passive Uncertainties}

Having established an appropriate framework for the propagation of uncertainty, we will now consider the estimation of the required active and passive uncertainties in the necessary form, that is, the complex bivariate covariance matrices, $\boldsymbol{\Sigma}_{\mathbf{v}}$ and $\boldsymbol{\Sigma}_{\mathbf{Z}}$. 


\subsection{Velocity Uncertainties (Active)}

The complex bivariate covariance matrix, $\boldsymbol{\Sigma}_{\mathbf{v}} \in \mathbb{R}^{2 N \times 2 N}$, may be determined with relative ease through the independent and repeated operation of the source. However, care must be taken when dealing with rotating machinery, particularly when tonal components are present. In such a case, consecutive time windows of a single operational run would see a large periodic uncertainty in the velocity, due to the window gradually moving through the phase cycle of the tonal component. This may be avoided, however, through the use of an appropriate phase reference.

\subsection{Impedance Uncertainties (Passive)}

The uncertainty propagation framework established in section 4 requires knowledge of the uncertainties associated with the impedance matrix, $\mathbf{Z} \in \mathbb{C}^{M \times N}$, in the form of its complex bivariate covariance matrix, $\boldsymbol{\Sigma}_{\mathbf{Z}} \in \mathbb{R}^{2 M N \times 2 M N}$. However, impedances are not generally measured directly due to the impracticality of applying the necessary constraints. Instead, it is common to measure mobilities matrices, $\mathbf{Y} \in \mathbb{C}^{N \times M}$ which may be inverted to obtain impedance. We are therefore concerned with the estimation of impedance uncertainty through the measurement of mobility, i.e. the propagation of uncertainty through a matrix inversion.

In the measurement of mobility it is standard practice to perform repeated measurements, taking their average as the 'true' value. Typically this is an exercise to reduce the effect of background noise, and little to no use is made of the individual measurements. However, with the determination of an appropriate mobility covariance matrix in mind, repeated measurements may be used to provide statistical information regarding the uncertainty of the measured mobilities. Whilst there exist established formulas for the estimation of mobility variance as a function of its expected value and associated coherence [22], these are based on the assumption of uncorrelated noise, which will not be valid in the case of operator uncertainty (due to inconsistent force excitations). For this reason we consider a more general approach. Suppose we wish to estimate the uncertainty of the mobility matrix $\mathbf{Y}$. The $M$ excitations (i.e. the columns of the mobility matrix) are each repeated $P$ times (i.e. we excite each DoF $P$ times such that we have $P$ 'realisations' of each matrix column). The resultant mobilities are arranged such that,

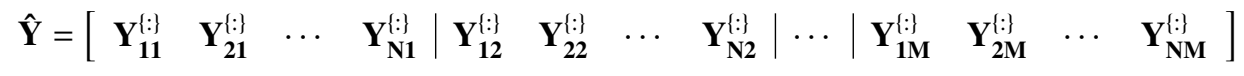

where, $\hat{\mathbf{Y}} \in \mathbb{R}^{P \times 2 N M}$ is used to denote the reordered mobility matrix, $M$ and $N$ correspond to row and column indices, respectively, and $\mathbf{Y}_{\mathrm{ij}}^{[:\}}=\left[\mathfrak{R}\left(\mathbf{Y}_{\mathrm{ij}}^{[:\}}\right) \quad \mathfrak{J}\left(\mathbf{Y}_{\mathrm{ij}}^{[:\}}\right)\right] \in \mathbb{R}^{P \times 2}$ contains the real and imaginary components of the $P$ measurements (or 'realisations') of $Y_{i j}$. The above is clearly amenable to the estimation of the complex bivariate covariance matrix, $\boldsymbol{\Sigma}_{\mathbf{Y}} \in \mathbb{R}^{2 N M \times 2 N M}$.

In the construction of $\boldsymbol{\Sigma}_{\mathbf{Y}}$ the existence of a meaningful covariance between particular elements of the mobility matrix must be considered. The elements of a given column of the mobility matrix are clearly correlated through their shared excitation [32]. Neighbouring columns, however, are likely to be uncorrelated owing to their independent excitations. As such, the covariance matrix $\boldsymbol{\Sigma}_{\mathbf{Y}}$ should be block diagonal, i.e. the mobilities due to an excitation at DoF $i$ are uncorrelated to those due to an excitation at $\operatorname{DoF} j$, for $i \neq j$.

It is important to note that when estimating the covariance matrix $\boldsymbol{\Sigma}_{\mathbf{Y}}$, as per the above procedure, one acquires a measure of the uncertainty between the mobilities of independent excitations. It may be argued that a more representative measure of uncertainty would be for that of the mean value, $\mathbb{E}[\mathbf{Y}]$. This is readily found by normalising $\boldsymbol{\Sigma}_{\mathbf{Y}}$ by the number of excitations applied, $P$. In doing so $\Sigma_{\mathbf{Y}} / P$ would represent an estimate of the uncertainty in $\mathbb{E}[\mathbf{Y}]$. This normalisation does not effect the formulation of the proposed framework, nor any later developments presented in this work. The normalised covariance matrix is simply used in place of $\boldsymbol{\Sigma}_{\mathbf{Y}}$. The results presented through section 6 however do not include this normalisation factor, and therefore represent the uncertainty related to results obtained from a single set of excitations. Its introduction would simply reduce the level of uncertainty by a constant factor.

Having estimated the uncertainty of the mobility matrix, we are interested in establishing the uncertainty of the impedance matrix, $\mathbf{Z}=\mathbf{Y}^{\mathbf{- 1}}$, or $\mathbf{Z}=\mathbf{Y}^{+}$in the case of a non-square mobility matrix. In general, the inversion of a matrix is a non-linear process. As such, there is no clear mapping between the uncertainties, $\boldsymbol{\Sigma}_{\mathbf{Y}}$ and $\boldsymbol{\Sigma}_{\mathbf{Z}}$. A similar problem was encountered by Ackerstaff et al. [49] concerning the decay of the tau lepton. A simplified approach was proposed where only diagonal uncertainties were considered, i.e. it was assumed that there existed no correlation between the matrix elements. An extension was later provided to account for inter-element correlation, where an inverse propagation formula was established [50]. This formula was further extended by Meggitt [32] for complex 
square matrices. In what follows we will derive a generalised inverse propagation formula, valid for real and complex valued, square and non-square matrices.

\subsubsection{Propagation of Mobility Uncertainty Through a Matrix Inversion}

We begin by considering the measured mobility matrix $\mathbf{Y} \in \mathbb{C}^{N \times M}$, whose uncertainty is characterised by the bivariate covariance matrix $\Sigma_{\mathbf{Y}} \in \mathbb{R}^{2 N M \times 2 N M}$. Noting that an arbitrary element of the bivariate impedance covariance matrix $\boldsymbol{\Sigma}_{\mathbf{Z}} \in \mathbb{R}^{2 N M \times 2 N M}$ is given by,

$$
\boldsymbol{\Sigma}_{\mathbf{Z}_{\mathbf{i j}}, \mathbf{Z}_{\mathbf{l m}}}=\left[\begin{array}{cc}
\sigma_{\mathfrak{R}\left(Z_{i j}\right) \mathfrak{R}\left(Z_{l m}\right)} & \sigma_{\mathfrak{R}\left(Z_{i j}\right) \mathfrak{I}\left(Z_{l m}\right)} \\
\sigma_{\mathfrak{I}\left(Z_{i j}\right) \mathfrak{R}\left(Z_{l m}\right)} & \sigma_{\mathfrak{I}\left(Z_{i j}\right) \mathfrak{I}\left(Z_{l m}\right)}
\end{array}\right],
$$

we are interested in determining a first order relation between $\boldsymbol{\Sigma}_{\mathbf{Z}_{\mathrm{ij}}, \mathbf{Z}_{\mathrm{lm}}}$ and $\boldsymbol{\Sigma}_{\mathbf{Y}}$. Noting that mobility and impedance are inversely related, we are concerned with the propagation of uncertainty through a matrix inversion. For generality, we will consider propagation through the Moore-Penrose pseudo-inverse [45], such that complex non-square matrices may be accommodated.

As per the law of error propagation, a first order linear relationship between $\boldsymbol{\Sigma}_{\mathbf{Z}_{\mathrm{ij}}, \mathbf{Z}_{\mathbf{l m}}}$ and $\boldsymbol{\Sigma}_{\mathbf{Y}}$ is given by,

$$
\Sigma_{\mathbf{Z}_{\mathrm{ij}}, \mathbf{Z}_{\mathrm{lm}}}=\mathbf{J}_{\mathrm{ij}} \boldsymbol{\Sigma}_{\mathbf{Y}} \mathbf{J}_{\mathbf{l m}}^{\mathbf{T}}
$$

where $\mathbf{J}_{\mathbf{i j}}$ once again represents the Jacobian of the propagation function. Since we are considering separately the real and imaginary components of the impedance and mobility matrices, the Jacobian $\mathbf{J}_{\mathbf{i j}}$ is given as a matrix of partial derivatives with respect to the real and imaginary components of each element,

$$
\mathbf{J}_{\mathbf{i j}}=\left[\begin{array}{ccccc}
\frac{\partial \mathfrak{R}\left(Z_{i j}\right)}{\partial \mathfrak{R}\left(Y_{11}\right)} & \frac{\partial \mathfrak{R}\left(Z_{i j}\right)}{\partial \mathfrak{I}\left(Y_{11}\right)} & \cdots & \frac{\partial \mathfrak{R}\left(Z_{i j}\right)}{\partial \mathfrak{R}\left(Y_{N M}\right)} & \frac{\partial \mathfrak{R}\left(Z_{i j}\right)}{\partial \mathfrak{I}\left(Y_{N M}\right)} \\
\frac{\partial \mathfrak{I}\left(Z_{i j}\right)}{\partial \mathfrak{R}\left(Y_{11}\right)} & \frac{\partial \mathfrak{I}\left(Z_{i j}\right)}{\partial \mathfrak{I}\left(Y_{11}\right)} & \cdots & \frac{\partial \mathfrak{I}\left(Z_{i j}\right)}{\partial \mathfrak{R}\left(Y_{N M}\right)} & \frac{\partial \mathfrak{I}\left(Z_{i j}\right)}{\partial \mathfrak{I}\left(Y_{N M}\right)}
\end{array}\right]
$$

To evaluate the Jacobian, we begin by noting that the complex differential of a Moore-Penrose pseudo-inverse $\mathbf{Y}^{+}$may be expressed in terms of $\mathbf{Y}$, its pseudo-inverse $\mathbf{Y}^{+}$, and its complex conjugate $\mathbf{Y}^{*}$, in the form [51],

$$
d \mathbf{Y}^{+}=-\mathbf{Y}^{+}(d \mathbf{Y}) \mathbf{Y}^{+}+\mathbf{Y}^{+} \mathbf{Y}^{+\mathbf{H}}\left(d \mathbf{Y}^{\mathbf{H}}\right)\left(\mathbf{I}-\mathbf{Y} \mathbf{Y}^{+}\right)+\left(\mathbf{I}-\mathbf{Y}^{+} \mathbf{Y}\right)\left(d \mathbf{Y}^{\mathbf{H}}\right) \mathbf{Y}^{+\mathbf{H}} \mathbf{Y}^{+}
$$

where $^{\mathbf{H}}$ represents the Hermitian, or conjugate transpose operation. Note that since the differential depends explicitly on the conjugate $\mathbf{Y}^{*}$, the pseudo-inverse function is non-analytic. As such we are unable to rely on the CauchyRiemann equations to establish the appropriate derivatives. In the above, $d \mathbf{Y}^{+}$may be interpreted as the small (complex) change in $\mathbf{Y}^{+}$given a small change $d \mathbf{Y}$ in $\mathbf{Y}$ (and $d \mathbf{Y}^{*}$ in $\mathbf{Y}^{*}$, which are of course related through conjugation). If we consider the small change in $\mathbf{Y}$ to be confined to a single element, say $Y_{s t}$, then $d \mathbf{Y}$ will be 0 in all but the st entry. In this regard, $d \mathbf{Y}$ may be replaced by the scaled single entry matrix $d \mathbf{Y}=\mathbf{P}_{\mathbf{s t}}\left(\partial Y_{s t}\right)$, where the scalar partial derivative $\partial Y_{s t}$ is introduced. Similarly, $d \mathbf{Y}^{\mathbf{H}}$ may be replaced by $d \mathbf{Y}^{\mathbf{H}}=\left(d \mathbf{Y}^{*}\right)^{\mathbf{T}}=\left(\partial Y_{s t}^{*} \mathbf{P}_{\mathrm{st}}\right)^{\mathbf{T}}=\mathbf{P}_{\mathrm{ts}} \partial Y_{s t}^{*}$. Consequently, the above differential becomes,

$$
\partial \mathbf{Y}^{+}=\left[-\mathbf{Y}^{+} \mathbf{P}_{\mathbf{s t}} \mathbf{Y}^{+}\right] \partial Y_{s t}+\left[\mathbf{Y}^{+} \mathbf{Y}^{+\mathbf{H}} \mathbf{P}_{\mathbf{t s}}\left(\mathbf{I}-\mathbf{Y} \mathbf{Y}^{+}\right)+\left(\mathbf{I}-\mathbf{Y}^{+} \mathbf{Y}\right) \mathbf{P}_{\mathbf{t s}} \mathbf{Y}^{+\mathbf{H}} \mathbf{Y}^{+}\right] \partial Y_{s t}^{*}
$$

where the right hand brackets correspond, respectively, to the partial derivatives of $\mathbf{Y}^{+}$with respect to $\mathbf{Y}$ and its conjugate $\mathbf{Y}^{*}$,

$$
\begin{gathered}
\frac{\partial \mathbf{Y}^{+}}{\partial Y_{s t}}=-\mathbf{Y}^{+} \mathbf{P}_{\mathbf{s t}} \mathbf{Y}^{+} \\
\frac{\partial \mathbf{Y}^{+}}{\partial Y_{s t}^{*}}=\mathbf{Y}^{+} \mathbf{Y}^{+\mathbf{H}} \mathbf{P}_{\mathbf{t s}}\left(\mathbf{I}-\mathbf{Y} \mathbf{Y}^{+}\right)+\left(\mathbf{I}-\mathbf{Y}^{+} \mathbf{Y}\right) \mathbf{P}_{\mathbf{t s}} \mathbf{Y}^{+\mathbf{H}} \mathbf{Y}^{+} .
\end{gathered}
$$

Note that we are interested in the derivatives of $\mathfrak{R}\left(\mathbf{Y}^{+}\right)$and $\mathfrak{J}\left(\mathbf{Y}^{+}\right)$with respect to the real and imaginary components of $\mathbf{Y}$, not its complex conjugate. The appropriate derivatives are found by first taking the real part of the 
differential $d \mathbf{Y}^{+}$(denoting, for clarity, $\frac{\partial \mathbf{Y}^{+}}{\partial Y_{s t}}$ and $\frac{\partial \mathbf{Y}^{+}}{\partial Y_{s t}^{*}}$ as $\mathbf{A}$ and $\mathbf{B}$, respectively),

$$
\mathfrak{R}\left(\partial \mathbf{Y}^{+}\right)=\mathfrak{R}\left(\mathbf{A} \partial Y_{s t}\right)+\mathfrak{R}\left(\mathbf{B} \partial Y_{s t}^{*}\right) .
$$

Recalling that $\mathfrak{R}([a+i b][c+i d])=a c-b d$, equation 37 may be rewritten as,

$$
\mathfrak{R}\left(\partial \mathbf{Y}^{+}\right)=\left[\mathfrak{R}(\mathbf{A}) \mathfrak{R}\left(\partial Y_{s t}\right)-\mathfrak{J}(\mathbf{A}) \mathfrak{J}\left(\partial Y_{s t}\right)\right]+\left[\mathfrak{R}(\mathbf{B}) \mathfrak{R}\left(\partial Y_{s t}^{*}\right)-\mathfrak{J}(\mathbf{B}) \mathfrak{J}\left(\partial Y_{s t}^{*}\right)\right]
$$

Further noting that $\mathfrak{R}\left(\partial Y_{s t}^{*}\right)=\mathfrak{R}\left(\partial Y_{s t}\right)$ and $\mathfrak{J}\left(\partial Y_{s t}^{*}\right)=-\mathfrak{J}\left(\partial Y_{s t}\right)$, the differential $\mathfrak{R}\left(\partial \mathbf{Y}^{+}\right)$becomes,

$$
\mathfrak{R}\left(\partial \mathbf{Y}^{+}\right)=[\mathfrak{R}(\mathbf{A})+\mathfrak{R}(\mathbf{B})] \mathfrak{R}\left(\partial Y_{s t}\right)+[-\mathfrak{J}(\mathbf{A})+\mathfrak{J}(\mathbf{B})] \mathfrak{J}\left(\partial Y_{s t}\right)
$$

Consequently, we have that,

$$
\frac{\partial \mathfrak{R}\left(\mathbf{Y}^{+}\right)}{\partial \mathfrak{R}\left(Y_{s t}\right)}=\mathfrak{R}\left(\frac{\partial \mathbf{Y}^{+}}{\partial Y_{s t}}+\frac{\partial \mathbf{Y}^{+}}{\partial Y_{s t}^{*}}\right), \quad \frac{\partial \mathfrak{R}\left(\mathbf{Y}^{+}\right)}{\partial \mathfrak{J}\left(Y_{s t}\right)}=\mathfrak{J}\left(-\frac{\partial \mathbf{Y}^{+}}{\partial Y_{s t}}+\frac{\partial \mathbf{Y}^{+}}{\partial Y_{s t}^{*}}\right)
$$

Taking the imaginary part of the differential $d \mathbf{Y}^{+}$, and following a similar procedure as above we arrive at,

$$
\frac{\partial \mathfrak{I}\left(\mathbf{Y}^{+}\right)}{\partial \mathfrak{R}\left(Y_{s t}\right)}=\mathfrak{I}\left(\frac{\partial \mathbf{Y}^{+}}{\partial Y_{s t}}+\frac{\partial \mathbf{Y}^{+}}{\partial Y_{s t}^{*}}\right), \quad \frac{\partial \mathfrak{I}\left(\mathbf{Y}^{+}\right)}{\partial \mathfrak{I}\left(Y_{s t}\right)}=-\mathfrak{R}\left(-\frac{\partial \mathbf{Y}^{+}}{\partial Y_{s t}}+\frac{\partial \mathbf{Y}^{+}}{\partial Y_{s t}^{*}}\right)
$$

Defining the bivariate Jacobian element as so,

$$
M_{i j}^{s t}=\left[\begin{array}{ll}
\frac{\partial \mathfrak{R}\left(Z_{i j}\right)}{\partial \mathfrak{R}\left(Y_{s t}\right)} & \frac{\partial \mathfrak{R}\left(Z_{i j}\right)}{\partial \mathfrak{I}\left(Y_{s t}\right)} \\
\frac{\partial \mathfrak{T}\left(Z_{i j}\right)}{\partial \mathfrak{R}\left(Y_{s t}\right)} & \frac{\partial \mathfrak{I}\left(Z_{i j}\right)}{\partial \mathfrak{I}\left(Y_{s t}\right)}
\end{array}\right]=\left[\begin{array}{cc}
\mathfrak{R}\left(\frac{\partial \mathbf{Y}^{+}}{\partial Y_{s t}}+\frac{\partial \mathbf{Y}^{+}}{\partial Y_{s t}^{*}}\right)_{i j} & \mathfrak{I}\left(-\frac{\partial \mathbf{Y}^{+}}{\partial Y_{s t}}+\frac{\partial \mathbf{Y}^{+}}{\partial Y_{s t}^{*}}\right)_{i j} \\
\mathfrak{I}\left(\frac{\partial \mathbf{Y}_{s}^{+}}{\partial Y_{s t}}+\frac{\partial \mathbf{Y}^{+}}{\partial Y_{s t}^{*}}\right)_{i j} & -\mathfrak{R}\left(-\frac{\partial \mathbf{Y}^{+}}{\partial Y_{s t}}+\frac{\partial \mathbf{Y}^{+}}{\partial Y_{s t}^{*}}\right)_{i j}
\end{array}\right]
$$

the bivariate impedance covariance matrix $\Sigma_{\mathbf{Z}_{\mathrm{ij}}, \mathbf{Z}_{\mathrm{Im}}}$ is given by,

$$
\left[\begin{array}{cc}
\sigma_{\mathfrak{R}\left(Z_{i j}\right) \mathfrak{R}\left(Z_{l m}\right)} & \sigma_{\mathfrak{R}\left(Z_{i j}\right) \mathfrak{I}\left(Z_{l m}\right)} \\
\sigma_{\mathfrak{I}\left(Z_{i j}\right) \mathfrak{R}\left(Z_{l m}\right)} & \sigma_{\mathfrak{I}\left(Z_{i j}\right) \mathfrak{I}\left(Z_{l m}\right)}
\end{array}\right]=\left[\begin{array}{lll}
M_{i j}^{11} & \cdots & M_{i j}^{N M}
\end{array}\right] \Sigma_{\mathbf{Y}}\left[\begin{array}{c}
\left(M_{l m}^{11}\right)^{\mathbf{T}} \\
\vdots \\
\left(M_{l m}^{N M}\right)^{\mathbf{T}}
\end{array}\right] .
$$

Equations 35-36 and 42-43 are the main results of this section. Together they describe the Linear-Inverse-Propagation (LIP) of complex uncertainty through a Moore-Penrose pseudo-inverse. As such, they facilitate the construction of the required impedance covariance matrix $\boldsymbol{\Sigma}_{\mathbf{Z}}$. Note that the impedance terms $\mathbf{Z}=\mathbf{Y}^{+}$in equations 35 and 36 correspond to those of the true impedance and must be replaced by the expected values obtained through the inversion of an expected mobility matrix.

It is interesting to note, in the special case that $\mathbf{Y} \in \mathbb{R}^{N \times N}$ is a real square matrix, equations 33,35 and 42 reduce to,

$$
d \mathbf{Y}^{-1}=-\mathbf{Y}^{-1}(d \mathbf{Y}) \mathbf{Y}^{-\mathbf{1}}, \quad \frac{\partial \mathbf{Y}^{-1}}{\partial Y_{s t}}=-\mathbf{Y}^{-1} \mathbf{P}_{\mathbf{s t}} \mathbf{Y}^{-1}, \quad M_{i j}^{s t}=\frac{\partial Z_{i j}}{\partial Y_{s t}}=\left(-\mathbf{Y}^{-1} \mathbf{P}_{\mathbf{s t}} \mathbf{Y}^{-1}\right)_{i j} .
$$

Acknowledging the above, and rewriting equation 43 as an element by element summation yields,

$$
\operatorname{Cov}\left[Z_{i j}, Z_{l m}\right]=\sum_{a, b, c, d} Z_{i a} Z_{b j} Z_{l c} Z_{d m} \operatorname{Cov}\left[Y_{a b}, Y_{c d}\right]
$$

This is in exact agreement with that of [50]. As such, equation 43 may be considered a generalisation of equation 45 for the case of complex non-square matrices, and constitutes a novel finding of this work.

Based on the law of error propagation, the above formulae are valid to first order and larger uncertainties will require an alternative approach such as a Monte-Carlo (MC) propagation. A MC propagation would involve the repeated inversion of independent realisations of a mobility matrix, each built from different combinations of the 
measured mobilities. The resulting impedance matrices are subsequently used to estimate the covariance matrix, $\boldsymbol{\Sigma}_{\mathbf{Z}}$. For small systems the MC approach may be used to compute the inverse for all possible combinations. However, the number of inversions required scales according to $P^{M}$, which clearly becomes intractable for systems of a realistic order. One may instead opt for a randomly sampled sub-set of possible combinations in order to reduce computational effort. The separation of uncertainties in terms of their real and imaginary components may also be performed using the MC approach by considering separately the statistics of the real and imaginary parts of the acquired impedance matrices.

The incorporation of equation 43, or the MC approach described above, within the propagation framework outlined by equations 24 - 27 forms the main contribution of this paper. Note however, the approximation introduced through the LIP or MC procedure will in turn lead to an uncertainty in the estimates provided by the framework. As such, care should be taken to ensure that a reliable $\boldsymbol{\Sigma}_{\mathbf{Z}}$ is obtained. In contrast to a standard MC simulation (where the entire inverse problem is solved repeatedly), the proposed framework may provide valuable insight regarding the contribution of each uncertain parameter (i.e. an element of the mobility matrix or velocity vector) to the uncertainty in the blocked force estimate. This may be beneficial, for example, in a benefit analysis where interest would lie in reducing the uncertainty in the blocked force by performing additional or repeated measurements.

As a final remark, although formulated in the context of force identification, the proposed framework makes no assumption regarding its application other than that the problem is of the form $\mathbf{x}=\mathbf{A b}$. As such, it may be applied to other inverse problems of this form, for example, the inverse characterisation of in-duct acoustic sources [52].

In the following section the proposed framework will be used to establish the uncertainties associated with inversely determined forces as part of 2 illustrative examples.

\section{Case Studies}

In this section the inverse propagation of uncertainty will be demonstrated via two examples. The first example, presented in section 6.1, concerns the reconstruction of externally applied forces on a numerical free-free beam model. The primary aim of this initial study is to validate the theoretical developments presented thus far. The second example, presented in section 6.2, considers the experimental determination of blocked forces and their use in the prediction of an operational response. This experimental study aims to illustrate an application of the proposed framework and demonstrates the estimation procedure.

\subsection{External Force Reconstruction}

Shown in figure 2 is a diagrammatic illustration of the numerical study considered here. A free-free beam is excited externally at two positions, labelled 1 and 2, each by a force-moment pair. The aim of this study is to reconstruct these external excitations using 'measured' mobilities and velocities and in doing so, provide a measure of the associated uncertainty. These measurements are simulated in such a way as to include the effect of both active and passive uncertainty.

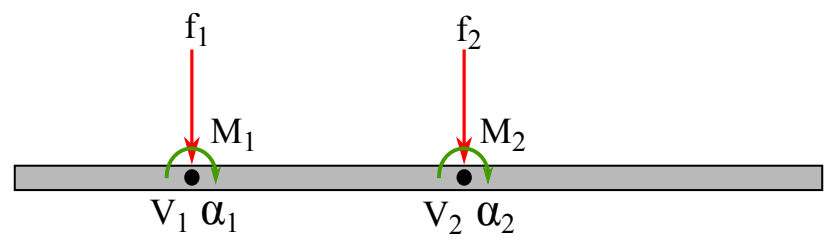

Figure 2: Diagrammatic illustration of numerical simulation. Operator uncertainty was modelled by varying the location of the applied forces and moments when calculating the FRFs.

The external force vector, $\mathbf{f}_{\mathbf{c}}^{\text {ext }}=\left[\begin{array}{llll}f_{1} & M_{1} & f_{2} & M_{2}\end{array}\right]^{T}$, is estimated through an inverse procedure, similar to that of the in-situ blocked force,

$$
\mathbf{f}_{\mathbf{c}}^{\mathrm{ext}}=\mathbf{Y}_{\mathrm{cc}}^{-1} \mathbf{v}_{\mathbf{c}}
$$

where; $\mathbf{v}_{\mathbf{c}} \in \mathbb{C}^{4}$ is an 'operational' velocity vector, and $\mathbf{Y}_{\mathbf{c c}} \in \mathbb{C}^{4 \times 4}$ is the associated mobility matrix. When measured, the above quantities are subject to a degree of uncertainty, as discussed through sections 2-3. The active uncertainty 
(i.e. in the velocity) is modelled here using a time domain noise model with a random Gaussian distributed amplitude. A total of 50 'measured' velocity vectors were acquired by summing the true velocity (given as the product of the input force vector and the true mobility matrix [see Appendix B]) to 50 independent and randomly generated noise vectors. These vectors are intended to simulate the cumulative effect of noise sources in the signal path (measurement uncertainty) and the stochastic behaviour of the external forces (source uncertainty). The passive uncertainty (i.e. in the mobility matrix) differs in that it is composed of measurement and operator uncertainty. For clarity, this study will consider only the effect of operator uncertainty on the measured mobilities. This was modelled using randomly distributed excitation positions, centred about positions 1 and 2. The position of each force and moment excitation was generated according to a Gaussian distribution. A total of 10 excitations were applied at each DoF. In keeping with experimental practice, response positions were kept fixed. The degree of operator uncertainty was set by the standard deviation of the excitation distribution, $\sigma$.

\begin{tabular}{cccccc}
$L \times W \times H$ & $\rho$ & $E$ & $\eta$ & $x_{j}$ & $x_{i}$ \\
\hline \hline $0.8 \times 0.1 \times 0.01$ & 7800 & $200 \mathrm{e} 9$ & 0.05 & $\mathcal{N}\left([0.3,0.6], \sigma^{2}\right)$ & {$[0.3,0.6]$}
\end{tabular}

Table 3: Model parameters; $L \times W \times H$ - dimensions, $\rho$ - density, $E$ - Young's modulus, $\eta$ - loss factor, $x_{j}$ - excitation position $\left(\mathcal{N}\left(\mu, \sigma^{2}\right)\right.$ represents a normal distribution of mean $\mu$ and standard deviation $\sigma), x_{i}$ - response position.

The material properties and geometry of the numerical model considered here are given in table 3 . The amplitudes of the input force vector were chosen to be real with arbitrary values of; $f_{1}=20, M_{1}=40, f_{2}=60$ and $M_{2}=80$.

The velocity and mobility uncertainties were estimated, as per section 5, in the form of their complex bivariate covariance matrices. The 50 velocity vectors returned the complex bivariate covariance matrix $\boldsymbol{\Sigma}_{\mathbf{v}} \in \mathbb{R}^{8 \times 8}$. Similarly, the repeated mobility measurements yielded the complex bivariate covariance matrix $\boldsymbol{\Sigma}_{\mathbf{Y}} \in \mathbb{R}^{32 \times 32}$. Shown in figure 3 are some exaggerated (to make clear the nature of the uncertainty) examples of the measurements from which these uncertainties were estimated. The operational uncertainty corresponds to a signal-to-noise ratio (SNR) of 20dB, and an operator uncertainty of $\sigma=10^{-3} \mathrm{~m}$. Establishing a representative level of uncertainty is not straightforward as it depends on: the nature of the structure under investigation, the experimental conditions (i.e. amount of access when performing the measurements), and the skill of the experimenter. For this reason a range of uncertainty levels are investigated, including $\sigma=10^{-5}, 10^{-4}$ and $10^{-3} \mathrm{~m}$, which encompass the range of values likely to occur in practice.

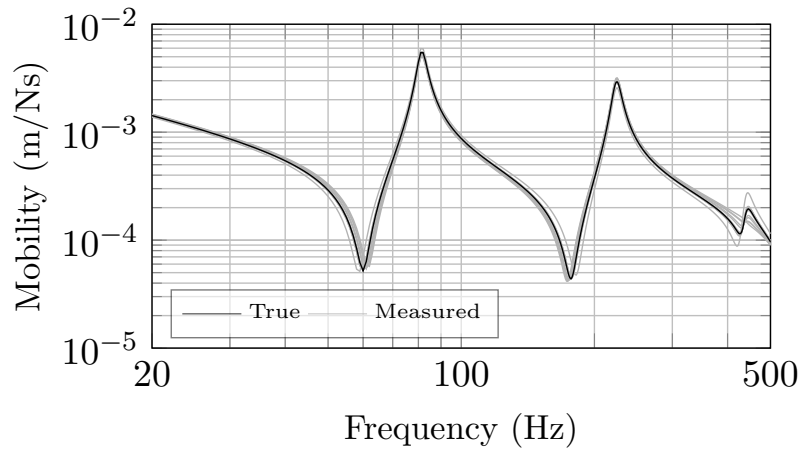

(a) True and 'measured' point mobilities $Y_{f_{1} v_{1}}$ with an uncertainty of $\sigma=0.01$.

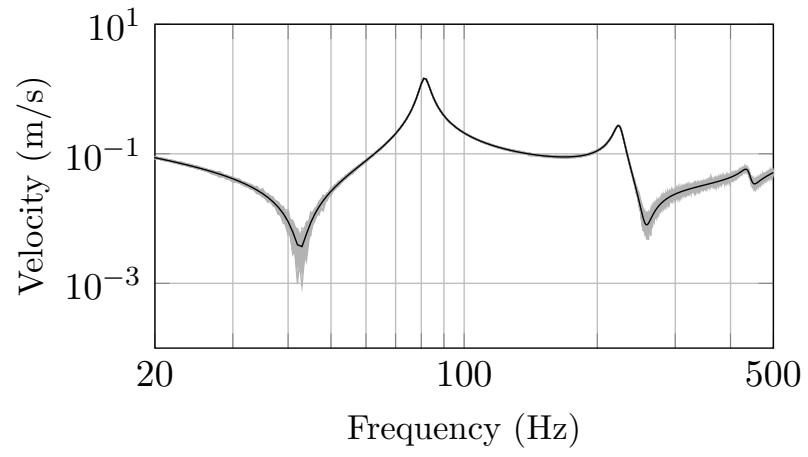

(b) True and 'measured' operational velocities $v_{1}$ with an SNR of $20 \mathrm{~dB}$

Figure 3: Example of true and virtually measured mobilities and velocities. Virtually measured mobilities are affected by human uncertainty (i.e. random excitation position), and measured velocities are affected by measurement uncertainty (i.e. noise in the signal path). Black line - true value, grey lines - uncertain measurements.

In order to propagate the above uncertainties through equation 46 one must first propagate the mobility uncertainty through the inversion process and onto the impedance. Two approaches were outlined in section 5, the first a Linear Inverse Propagation (LIP) method based on the law of error propagation and the complex differential of the MoorePenrose pseudo inverse, and the second, a MC approach, where a random ensemble of matrix permutations are inverted and the statistics of the resulting distribution analysed. Shown in figure 4 is a comparison of the two approaches for a 
complex square matrix with an operator uncertainty of $\sigma=10^{-5} \mathrm{~m}$. The relative variance of the real and imaginary components of the mobility element $Y_{11}$ and the impedance element $Z_{11}$ are shown in figures $4 \mathrm{a}$ and $4 \mathrm{~b}$, respectively. Shown in figure $4 \mathrm{c}$ is the relative covariance, defined here as,

$$
\operatorname{Rel} \operatorname{Cov}[A, B]=\frac{\sigma_{A B}}{\mathbb{E}[A] \mathbb{E}[B]}
$$

between the real and imaginary components of the same mobility and impedance elements. Lastly, figure $4 \mathrm{~d}$ shows the relative variance between the mobility and impedance magnitudes. In the case of the LIP prediction, this was calculated by first propagating the complex bivariate uncertainty through the matrix inversion, and then the magnitude function, as per equation 28. The MC prediction was acquired by taking the magnitude of all impedance realisations prior to calculating their variance.

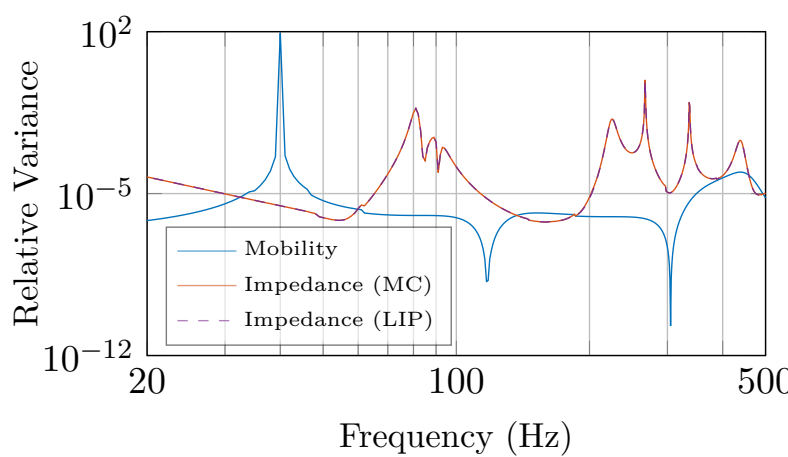

(a) Relative variance in the real part of the point mobilty and impedance, $\operatorname{RelVar}\left\{\mathfrak{R}\left(Y_{11}\right)\right\}$ and $\operatorname{RelVar}\left\{\mathfrak{R}\left(Z_{11}\right)\right\}$.

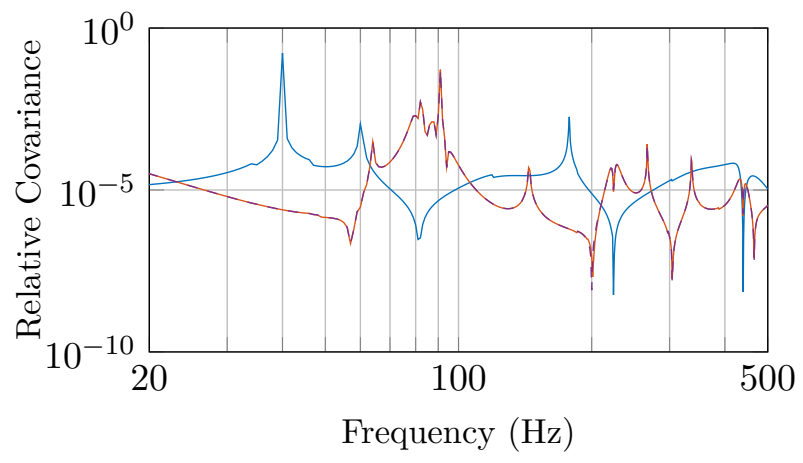

(c) Relative covariance between the real and imaginary parts of the point mobilty and impedance, $\operatorname{RelCov}\left\{\mathfrak{R}\left(Y_{11}, \mathfrak{J}\left(Y_{11}\right)\right)\right\}$ and $\operatorname{RelCov}\left\{\mathfrak{R}\left(Z_{11}\right), \mathfrak{J}\left(Z_{11}\right)\right\}$.

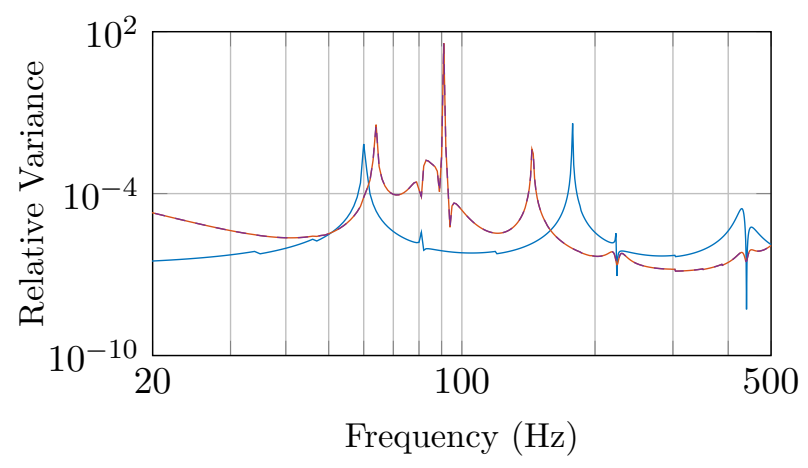

(b) Relative variance in the imaginary part of the point mobilty and impedance, $\operatorname{RelVar}\left\{\mathfrak{J}\left(Y_{11}\right)\right\}$ and $\operatorname{RelVar}\left\{\mathfrak{J}\left(Z_{11}\right)\right\}$.

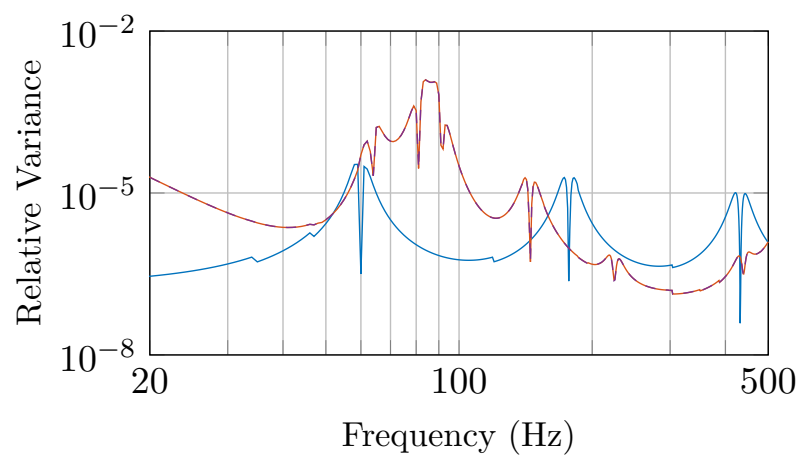

(d) Relative variance in the magnitude of the point mobilty and impedance, $\operatorname{RelVar}\left\{\left|Y_{11}\right|\right\}$ and RelVar$\left\{\left|Z_{11}\right|\right\}$.

Figure 4: Numerical simulation of the uncertainty propagation through matrix inversion using Monte-Carlo (MC) and Linear Inverse Propagation (LIP) methods for an experimental uncertainty $\sigma=10^{-5} \mathrm{~m}$. The relative variance shown for the real and imaginary parts and magnitude of mobility $Y_{11}$ and impedance $Z_{11}$. The relative covariance, defined here as, $\operatorname{Rel} \operatorname{Cov}\{A, B\}=\frac{\sigma_{A B}}{\mathbb{E}[A] \mathbb{E}[B]}$, is also shown between the real and imaginary components of $Y_{11}$ and $Z_{11}$.

Figure 4 illustrates; 1) that there exists no clear mapping between the uncertainty in the real and imaginary components (or the magnitude) of the mobility and impedance, and 2) that for a small level of uncertainty $\left(\sigma=10^{-5} \mathrm{~m}\right)$ the LIP method offers an accurate propagation of the mobility uncertainty, as illustrated by its agreement with the MC prediction. The results of figure 4 are replicated in figure 5 for an increased operator uncertainty of $\sigma=10^{-3} \mathrm{~m}$. This increase in the level of uncertainty clearly worsens the agreement between LIP and MC predictions. This is a consequence of the linear approximation made by the LIP approach. In such a case it is the MC propagation that 
should be considered the more reliable of the two.

Having acquired the impedance uncertainty, $\mathbf{\Sigma}_{\mathbf{Z}} \in \mathbb{R}^{32 \times 32}$, we are able to complete the inverse uncertainty propagation, as per equation 23. Shown in figure 6 are the magnitudes of the reconstructed forces acquired using the expected velocity vector and mobility matrix. Also shown are the $\pm 95 \%$ confidence intervals of the force estimates, acquired from the covariance matrix, $\boldsymbol{\Sigma}_{\mid \mathbf{f}}$, itself estimated as per equation 28 . These confidence intervals are presented for an assumed log-normal distribution following the formulation presented in Appendix C of [53]. Note that no normalisation was used when estimating the covariance matrix of the mobility. As such the confidence bounds presented in figure 6 represent the likelihood of predicting a blocked force from a single set of mobilities (i.e. without averaging multiple excitations).

The expected force reconstructions are in reasonable agreement with the true values, although some discrepancies are observed. These are likely a result of inconsistencies introduced by the operator uncertainty, the effects of which are amplified by the process of inversion. Nevertheless, the confidence intervals clearly encapsulate the true force, whilst illustrating the increased uncertainty associated with the disagreement in the predicted forces. For the operator uncertainty considered ( $\sigma=10^{-4} \mathrm{~m}$, a compromise between figures 4 and 5) the MC and LIP methods are also in good agreement, deviating only marginally in some regions.

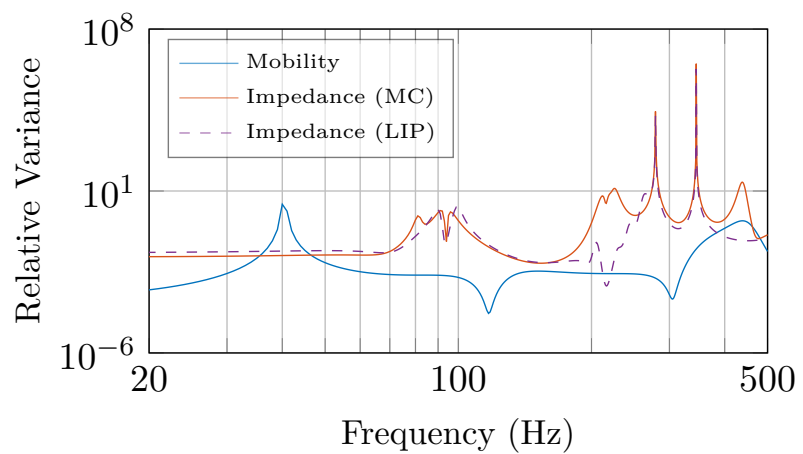

(a) Relative variance in the real part of the point mobilty and impedance, $\operatorname{RelVar}\left\{\mathfrak{R}\left(Y_{11}\right)\right\}$ and $\operatorname{RelVar}\left\{\mathfrak{R}\left(Z_{11}\right)\right\}$

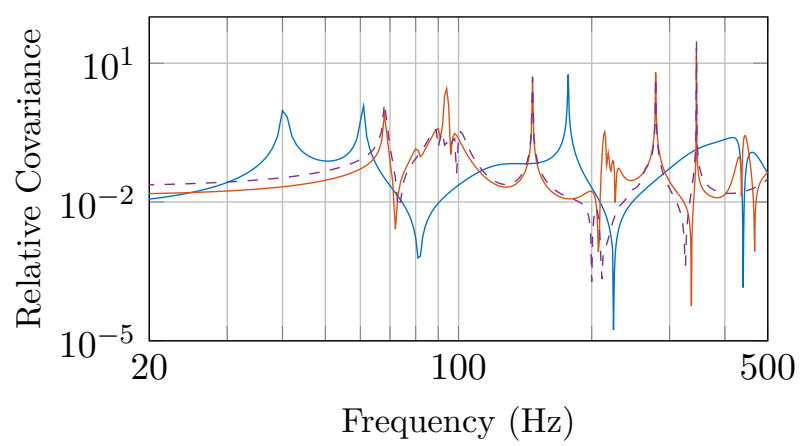

(c) Relative covariance between the real and imaginary parts of the point mobilty and impedance, $\operatorname{RelCov}\left\{\mathfrak{R}\left(Y_{11}, \mathfrak{J}\left(Y_{11}\right)\right)\right\}$ and $\operatorname{RelCov}\left\{\mathfrak{R}\left(Z_{11}\right), \mathfrak{J}\left(Z_{11}\right)\right\}$.

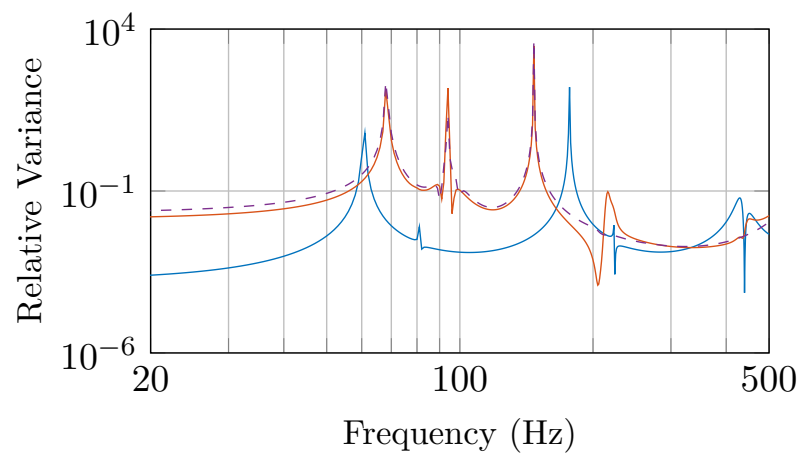

(b) Relative variance in the imaginary part of the point mobilty and impedance, $\operatorname{RelVar}\left\{\mathfrak{J}\left(Y_{11}\right)\right\}$ and $\operatorname{RelVar}\left\{\mathfrak{J}\left(Z_{11}\right)\right\}$.

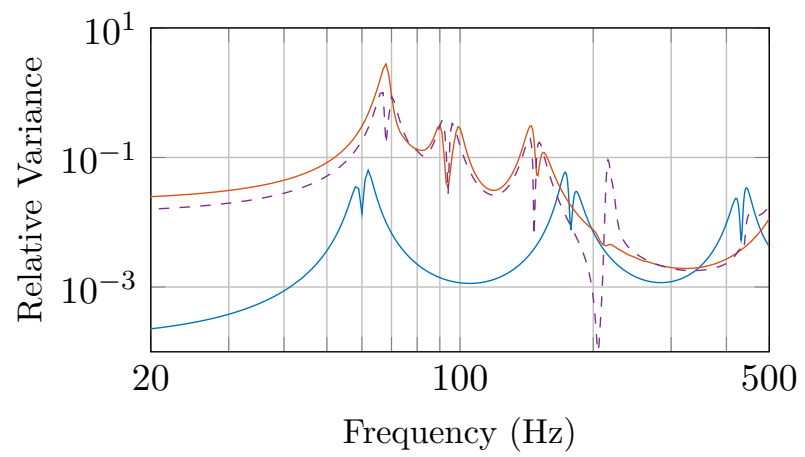

(d) Relative variance in the magnitude of the point mobilty and impedance, $\operatorname{Rel} \operatorname{Var}\left\{\left|Y_{11}\right|\right\}$ and $\operatorname{RelVar}\left\{\left|Z_{11}\right|\right\}$.

Figure 5: As figure 4, with an experimental uncertainty of $\sigma=10^{-3} \mathrm{~m}$.

As a final validation of the proposed framework, the estimated uncertainties are compared against those acquired using a full MC propagation. The full MC propagation required solving 50000 inverse problems (corresponding to every possible combination of mobility matrix realisations and noisy velocity vectors) at every frequency line. Such an approach clearly becomes intractable for problems of a realistic order, however, for the simulation considered the computational effort was not unreasonable. The resulting solutions were used to estimate the relative variance of the real and imaginary components, as well as their relative covariance (defined as per equation 47). The results are 
shown in figure 7 for an operator uncertainty of $\sigma=10^{-4} \mathrm{~m}$ and an SNR of $30 \mathrm{~dB}$. The two results presented for the proposed framework (denoted 'Cov propagation' in the figure legend) differ in that impedance covariance matrix was acquired either through a MC inversion (blue), or the LIP procedure (orange) outlined in section 5.2.1. Figure 7 illustrates, through agreement with the full MC prediction, that for the chosen levels of uncertainty $\left(\sigma=10^{-4} \mathrm{~m}\right.$ and an SNR (velocity) of $30 \mathrm{~dB}$ ), the proposed framework predicts accurately the uncertainty in the acquired force, in terms of both its real and imaginary components. In the case of LIP prediction (orange) some small deviations can be observed in the region of 90 and $200 \mathrm{~Hz}$. Such deviations would be expected given the approximations involved in the formulation of the LIP relation. Nevertheless, its agreement with the MC prediction provides a further validation of its formulation and application. It is worth noting that the estimated uncertainty using the MC inversion (blue) remains in good agreement with the full MC propagation. This suggests that in the presence of large uncertainty, although the LIP procedure may no longer be reliable, an MC inversion may still be used to accurately estimate the uncertainty in the acquired forces.

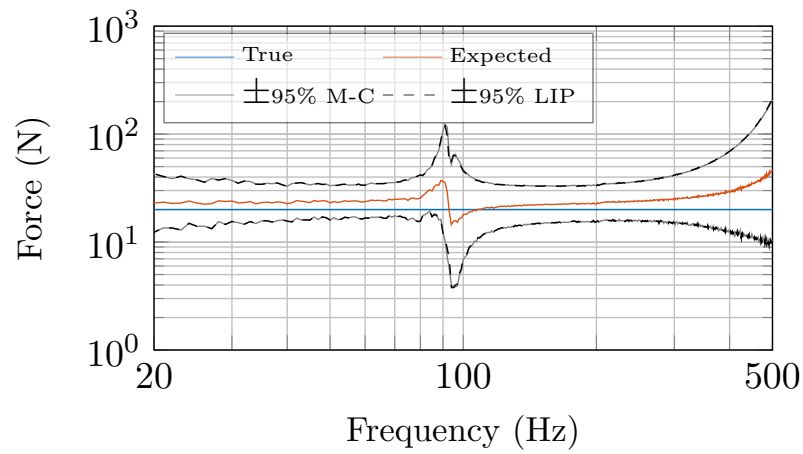

(a) $F_{1}$

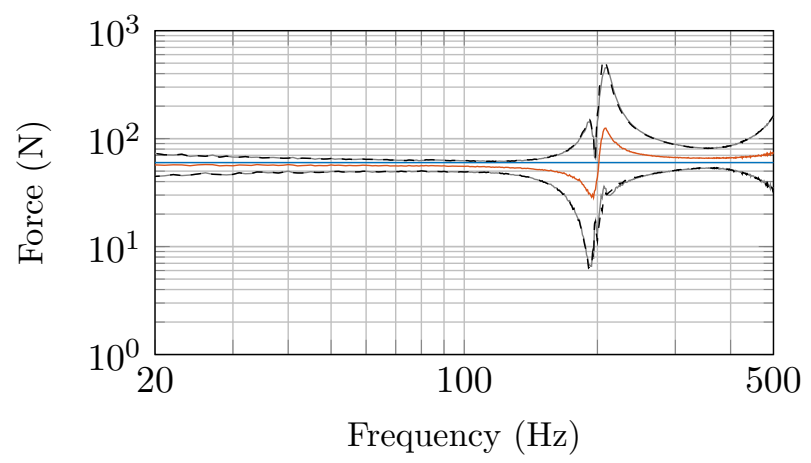

(c) $F_{2}$

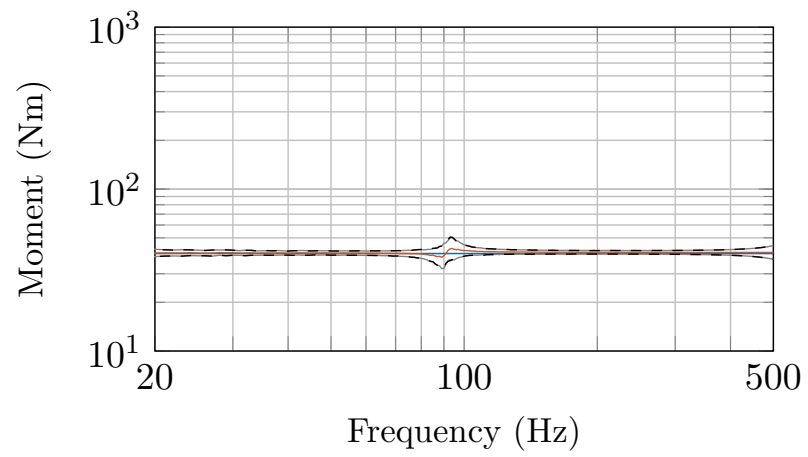

(b) $M_{1}$

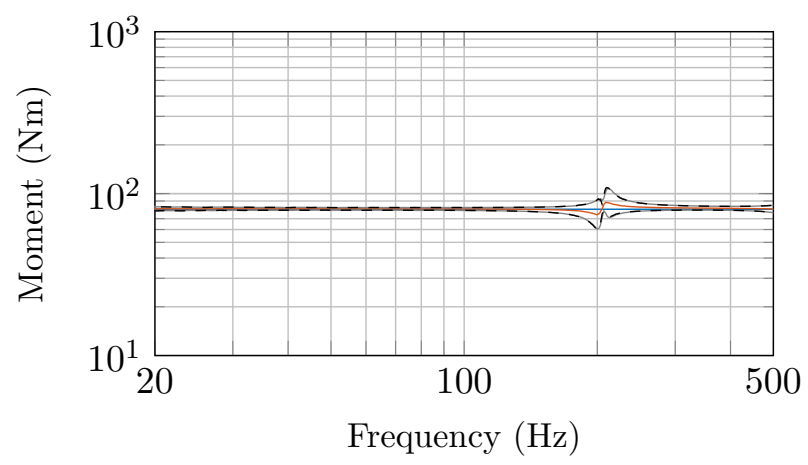

(d) $M_{2}$

Figure 6: Reconstructed forces using inverse approach with propagated uncertainties. Simulations were made with a passive uncertainty (human only) corresponding to $\sigma=10^{-4} \mathrm{~m}$ together with an active uncertainty (measurement only) corresponding to a signal to noise ratio of $30 \mathrm{~dB}$. $\pm 95 \%$ confidence intervals are presented for both LIP and MC inverse propagation approaches.

The numerical study considered above provides a validation of the proposed uncertainty framework, and illustrates its application. In the following section a similar procedure will be used to estimate the uncertainties associated with experimentally determined blocked forces, and subsequently propagate them through the prediction of an operational response. 


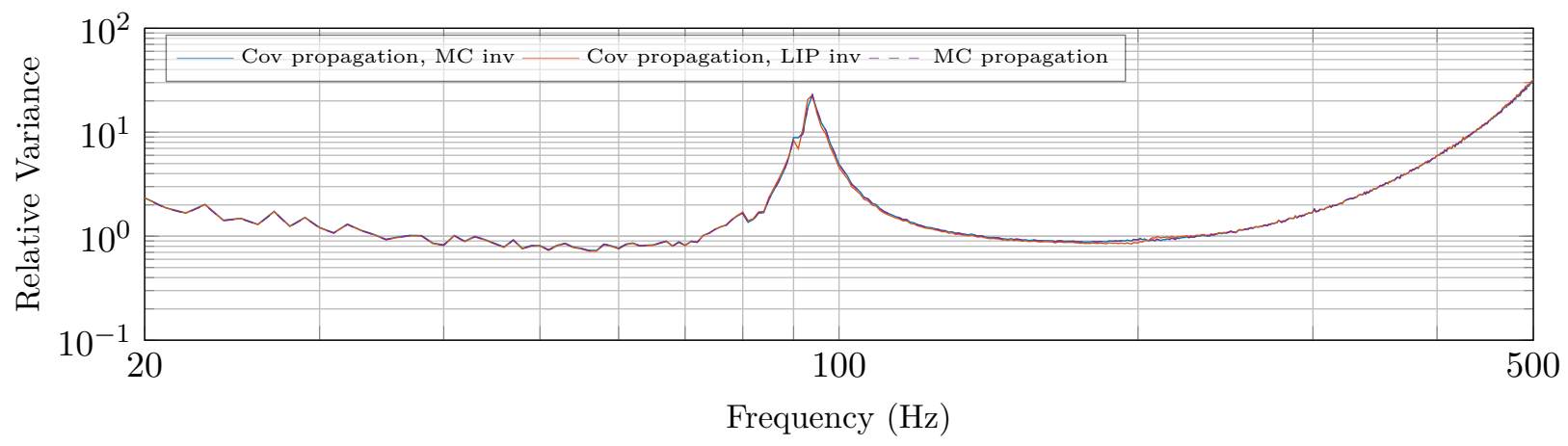

(a) Relative variance in the real part of the external force, $\operatorname{RelVar}\left\{\mathfrak{R}\left(f_{1}\right)\right\}$.
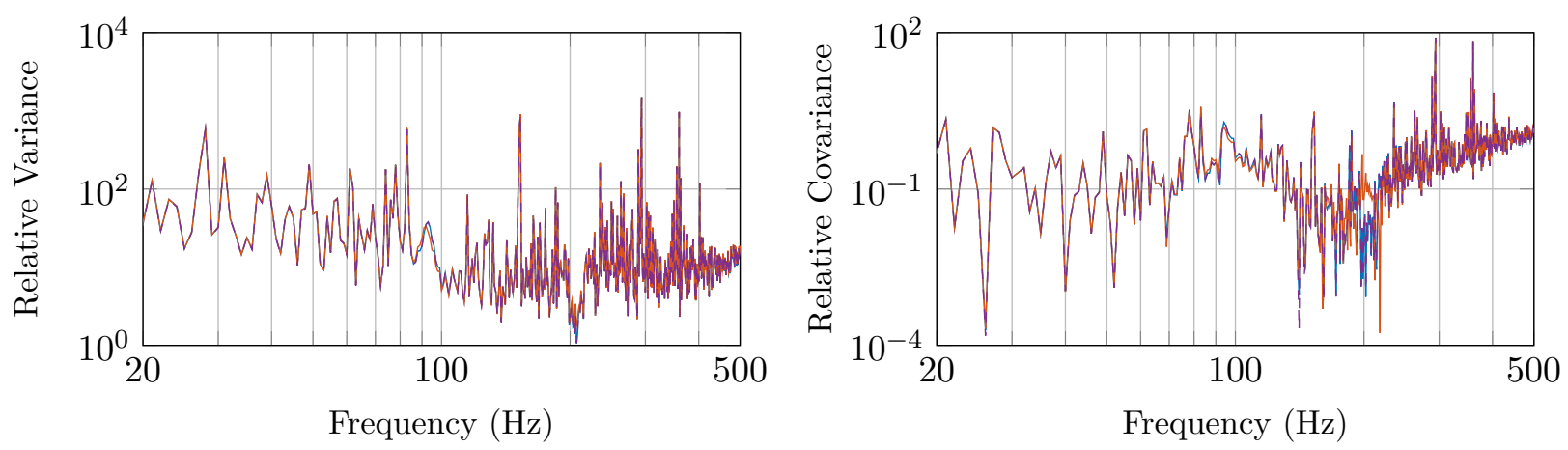

(b) Relative variance in the imaginary part of the external force, (c) Relative covariance between the real and imaginary parts of the exter-

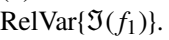
nal force, $\operatorname{RelCov}\left\{\mathfrak{R}\left(f_{1}\right), \mathfrak{J}\left(f_{1}\right)\right\}$.

Figure 7: A comparison of the estimated uncertainties based on the proposed framework (Cov) and a full Monte-Carlo (MC) simulation (purple). Cov propagation results are presented for the estimation of impedance uncertainty using both Monte-Carlo (blue) and Linear-Inverse-Propagation approaches (orange). Note that absolute values are shown to facilitate plotting on a logarithmic scale.

\subsection{In-situ Blocked Force Determination}

Shown in figure 8 is a diagrammatic representation of the experimental study considered here. A resiliently coupled source-receiver assembly (details of which are given in table 4) is excited externally (via impact) at a remote point on the source sub-structure. Here, the receiver sub-structure is considered to be that of the coupled resilient element-plate assembly. A resiliently coupled assembly was chosen to reduce the influence of rotational and in-plane DoFs, and thus minimize the effect of model uncertainty. For this reason, only out-of-plane translational DoFs are considered. To further avoid the introduction of rotational/in-plane DoFs, results are presented up to $1 \mathrm{kHz}$ only, beyond which translational DoFs are not sufficient in describing the interface dynamics.

The aims of this study are two fold: the first, to characterise the source sub-structure in terms of its blocked force and provide an estimate of the associated uncertainty; the second, once determined the blocked forces will be used to predict the operational response at a remote reference position, $\tilde{b}$, using the forward model,

$$
v_{\tilde{b}}=\mathbf{Y}_{\tilde{\mathbf{b}} \mathbf{c}} \overline{\mathbf{f}}_{\mathbf{c}}
$$

where the blocked force uncertainty is to be propagated through this response prediction so as to provide an estimate of the uncertainty, $\boldsymbol{\Sigma}_{v_{\tilde{b}}} \in \mathbb{R}^{2 \times 2}$. 


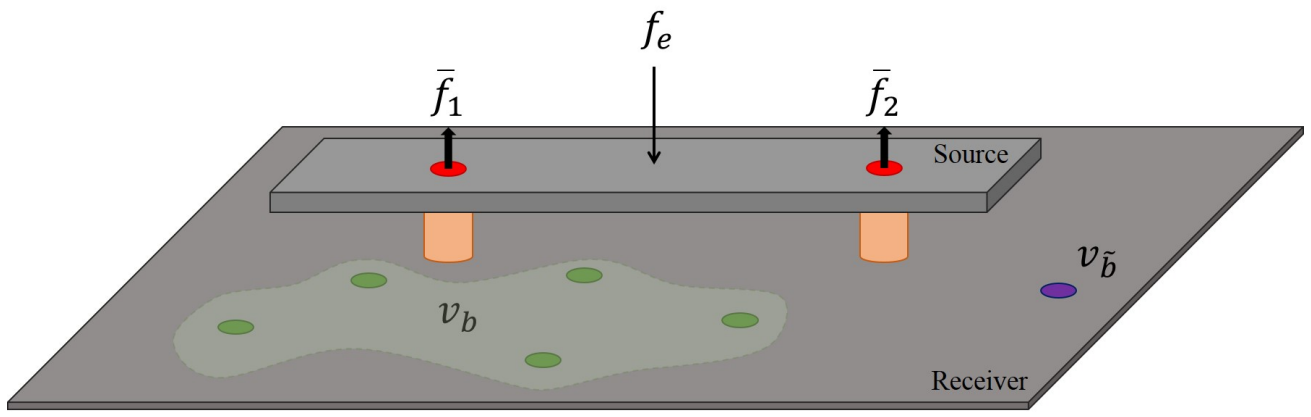

Figure 8: Diagrammatic representation of experimental study.

As with the blocked force, the uncertainty in the predicted operational velocity is found using the law of error propagation. The uncertainties associated with both the (active) blocked force and the (passive) forward mobility are considered, where it is further assumed that the two are uncorrelated. An appropriate forward propagation formula is obtained by modifying equations 23 and 25 such that the velocity $\mathbf{v}$ replaced by the blocked force $\overline{\mathbf{f}}_{\mathbf{c}}$, and the impedance $\mathbf{Z}$ by the forward mobility $\mathbf{Y}_{\tilde{\mathbf{b}} \mathbf{c}}$. The resultant expression is given in an expanded form by,

$$
\boldsymbol{\Sigma}_{\mathbf{v}_{\tilde{\mathbf{b}}}}=\left[\begin{array}{lll}
\ddots & & \\
& \overline{\mathbf{f}}_{\mathbf{c}} & \\
& & \ddots
\end{array}\right] \boldsymbol{\Sigma}_{\mathbf{Y}_{\tilde{\mathbf{b c}} \mathbf{c}}}\left[\begin{array}{lll}
\ddots & & \\
& \overline{\mathbf{f}_{\mathbf{c}}} & \\
& & \ddots
\end{array}\right]^{\mathbf{T}}+\mathbf{Y}_{\tilde{\mathbf{b}} \mathbf{c}} \boldsymbol{\Sigma}_{\overline{\mathbf{f}}} \mathbf{Y}_{\tilde{\mathbf{b}} \mathbf{c}}^{\mathbf{T}}
$$

where, $\boldsymbol{\Sigma}_{\mathbf{v}_{\tilde{b}}}$ is the complex bivariate covariance matrix of the remote reference response, $\left[\overline{\mathbf{f}}_{\mathbf{c}}\right] \boldsymbol{\Sigma}_{\mathbf{Y}_{\tilde{\mathbf{b}}}}\left[\overline{\mathbf{f}}_{\mathbf{c}}\right]^{\mathbf{T}}$ is the contribution of uncertainty due to the forward mobility, and $\mathbf{Y}_{\tilde{\mathbf{b}} \mathbf{c}} \boldsymbol{\Sigma}_{\overline{\mathbf{f}}} \mathbf{Y}_{\tilde{\mathbf{b}} \mathbf{c}}^{\mathbf{T}}$ the contribution due to the inversely determined blocked forces. Note that the blocked force uncertainty, $\boldsymbol{\Sigma}_{\overline{\mathbf{f}}}$, is itself a combination of the active and passive uncertainty present in the inverse procedure, i.e. $\boldsymbol{\Sigma}_{\overline{\mathbf{f}}}=\Sigma_{\overline{\mathbf{f}}_{\mathrm{Z}}}+\Sigma_{\overline{\mathbf{f}}_{\mathrm{v}}}$.

\begin{tabular}{c|cccc} 
& Type & Dimensions $(\mathrm{cm})$ & Material \\
\hline \hline Source & Beam & $5 \times 27 \times 0.9$ & $(w \times l \times h)$ & Steel \\
Receiver & Plate & $50 \times 40 \times 0.5$ & $(w \times l \times h)$ & Steel \\
Coupling element & Isolator & $1.2 \times 1.2$ & $(h \times \phi)$ & Rubber
\end{tabular}

Table 4: Details of the assembly used in the experimental study.

The blocked force, $\overline{\mathbf{f}}_{\mathbf{c}} \in \mathbb{C}^{2}$, was found by following the two part measurement procedure outlined in section 3 . Five remote measurement DoFs, $b$, were used in its determination, with an additional remote DoF, $\tilde{b}$, used to provide the on-board validation. Each mobility was measured 10 times, and the average used to construct the mobility matrix, $\mathbf{Y}_{\mathbf{b c}} \in \mathbb{C}^{5 \times 2}$. A 12 channel NetdB acquisition card was used, with all processing, bar FRF calculations, performed in MATLAB. All accelerometers where B\&K Type 4507 B 004 with nominal sensitivity $10 \mathrm{mV} / \mathrm{ms}^{-1}$. Note that no regularisation was used when performing the required matrix inversion.

Shown in figure $9 \mathrm{a}$ is an example of the spread obtained in the measurement of the mobility, $Y_{b_{1} c_{1}}$, alongside its expectation. Also shown (red circles) are the locations of the maximum condition number (shown explicitly in figure 10 ) in the expected mobility matrix, $\mathbf{Y}_{\mathbf{b c}}$. The individual mobility measurements were subsequently used to estimate the passive uncertainty in the form of the complex bivariate covariance matrix, $\boldsymbol{\Sigma}_{\mathbf{Y}} \in \mathbb{R}^{20 \times 20}$.

Shown in figure $9 \mathrm{c}$, in blue, is the relative variance of the transfer mobility magnitude, $\left|Y_{b_{1} c_{1}}\right|$. Peaks in the relative variance can be seen to largely coincide with the anti-resonant regions of the mobility, with resonances coinciding, generally, with regions of lower relative variance. This is an expected result as anti-resonances are dependent upon excitation position and therefore sensitive to human error, whilst the resonances are fixed in frequency according to geometrical and material properties. Although expected, this is an interesting observation as the peak condition numbers are seen to coincide with the resonant regions of low relative variance. The condition number is often 
assumed to indicate regions where inverse methods are susceptible to large uncertainties [38], so it is interesting to note that the variance (or experimental uncertainty) in the mobility is not related to ill conditioning.

The transient nature of the external force avoids the introduction of any operational source uncertainty, leaving only measurement uncertainty in the operational velocity, $\mathbf{v}_{\mathbf{b}} \in \mathbb{C}^{5}$. As a means simulating a more realistic scenario, artificial noise was added in post processing to simulate the effect of a random but stationary operational behaviour. Noise was introduced at an SNR of $25 \mathrm{~dB}$ using the approach discussed in section 6.1. Shown in figure 11 is an example of the artificially induced source uncertainty. From the 50 'measured' velocity vectors the complex bivariate covariance matrix, $\boldsymbol{\Sigma}_{\mathbf{v}} \in \mathbb{R}^{10 \times 10}$, was estimated.

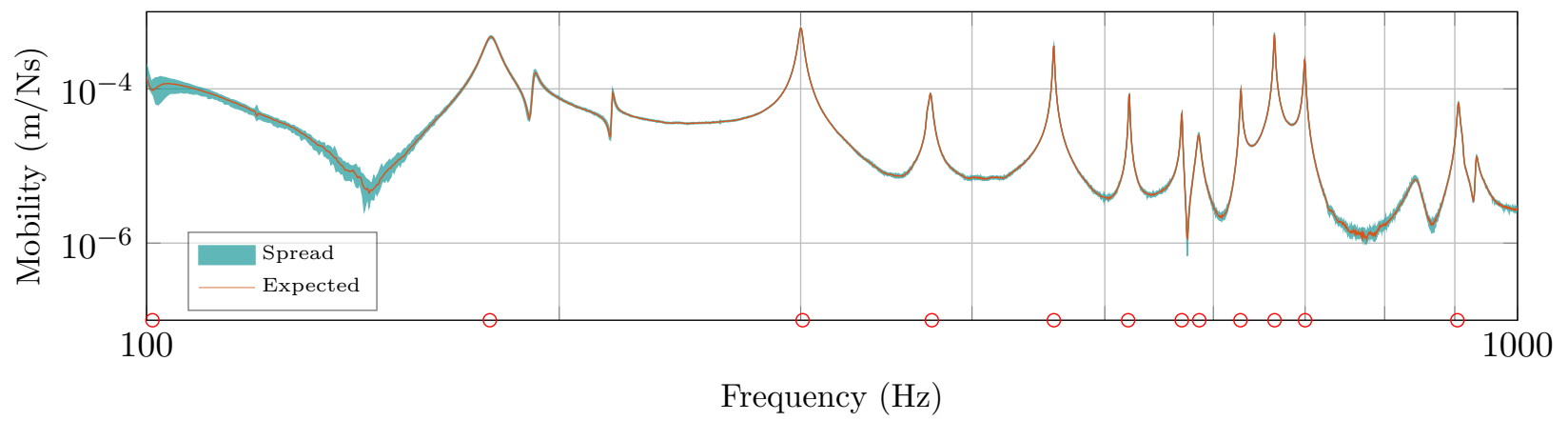

(a) Spread of transfer mobility, $Y_{b_{1} c_{1}}$.

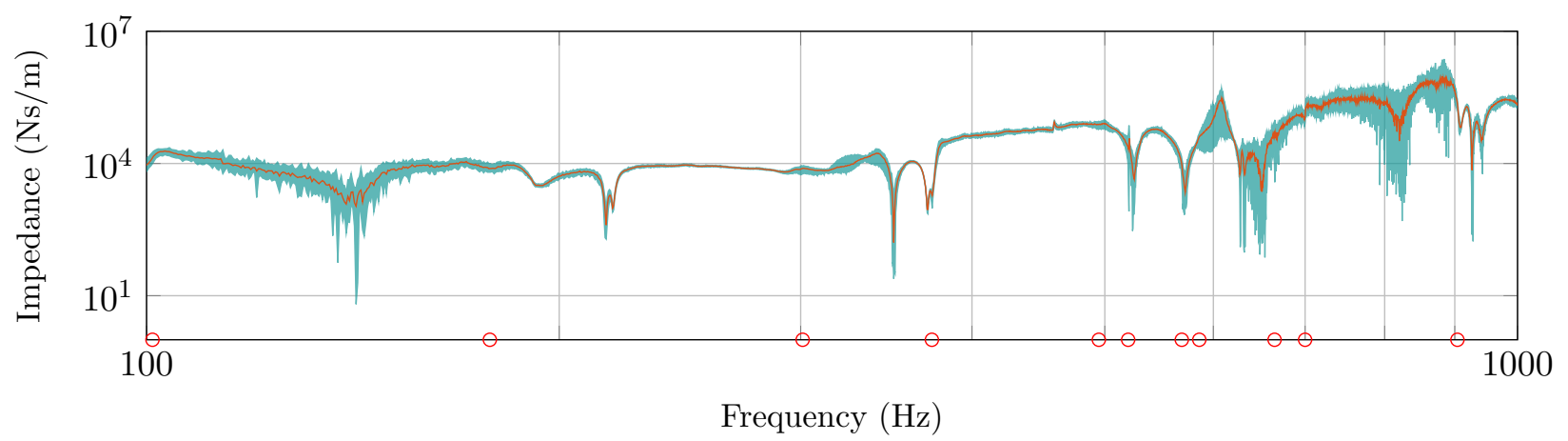

(b) Spread of transfer impedance, $Z_{b_{1} c_{1}}$.

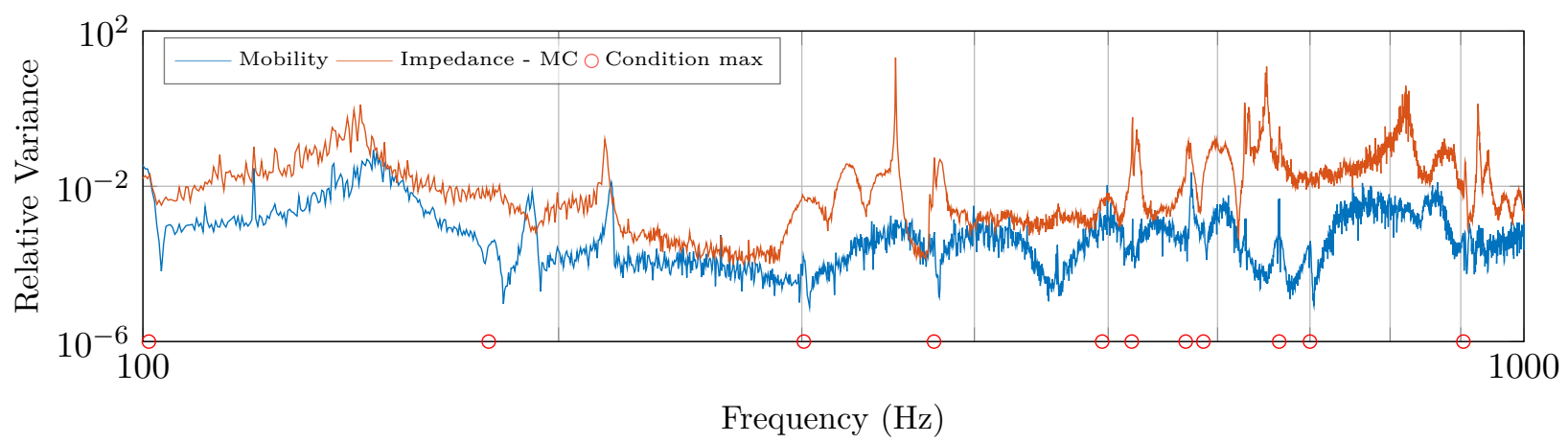

(c) Relative variance of transafer mobility and impedances, $Y_{b_{1} c_{1}}$ and $Z_{b_{1} c_{1}}$.

Figure 9: Expected value, spread, and relative variance of a measured transfer mobility, $Y_{b_{1} c_{1}}$, and corresponding inversely determined transfer impedance, $Z_{b_{1} c_{1}}$. Red circles correspond to peak values in the condition number of $\mathbf{Y}_{\mathbf{b c}}$.

The propagation framework outlined through section 4 requires an estimate of the impedance uncertainty, $\boldsymbol{\Sigma}_{\mathbf{Z}} \in$ 
$\mathbb{R}^{20 \times 20}$. Two approaches have been discussed to this end, the first a Linear Inverse Propagation (LIP), the second, a Monte-Carlo (MC) simulation. Shown in figure 12 is a comparison of these two methods for a complex nonsquare matirx. The bivariate uncertainty of the impedance element $Z_{b_{1} c_{1}}$ is considered. The MC simulation involved the inversion of 1000 randomly generated realisations of the mobility matrix. The agreement between the LIP and MC methods not only provides a further validation of the LIP relation in the presence of a non-square matrix, but demonstrates its applicability under a realistic level of experimental uncertainty.

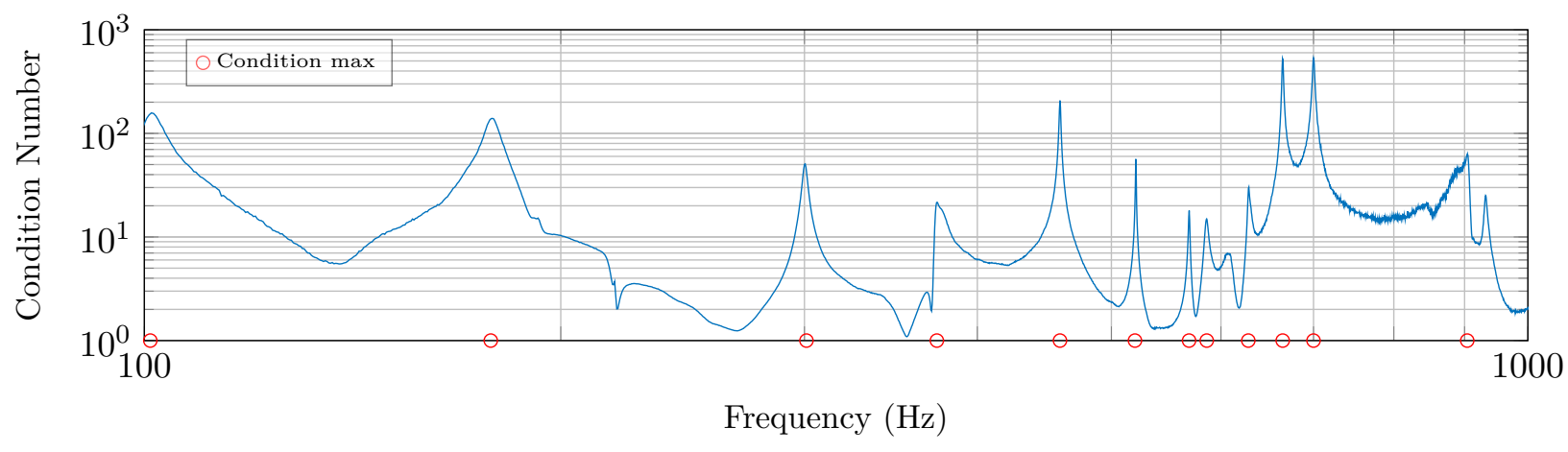

Figure 10: Condition number of the mobility matrix, $\mathbf{Y}_{\mathbf{c b}}$. Red circles correspond to regions of maximum condition number. These markers are carried through the remaining figures.

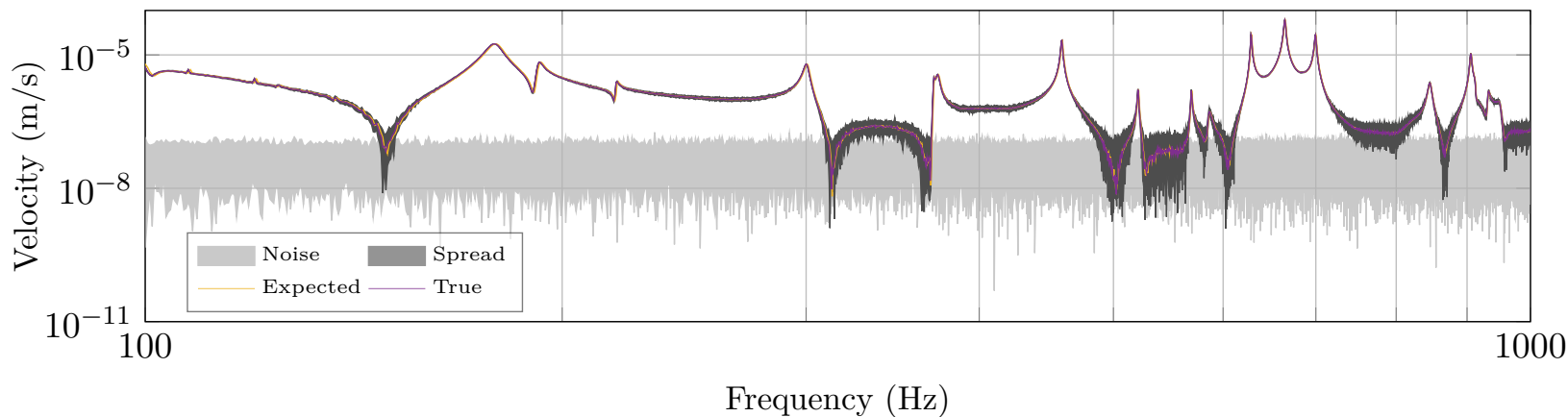

Figure 11: An example of the artificially induced source uncertainty on the measured operation velocity $v_{b_{1}}$. In light grey are the upper and lower bounds of the 50 noise vectors, in dark grey are the resulting uncertain velocities. The exact and expected velocities are shown in purple and yellow, respectively.

Shown in figure $9 \mathrm{~b}$ is the spread of the 1000 randomly generated realisations of the transfer impedance magnitude $\left|Z_{b_{1} c_{1}}\right|$. Shown alongside is the impedance obtained from inverting the expected mobility matrix. Shown in figure $9 \mathrm{c}$, in orange, is the relative variance of the impedance magnitude, $\left|Z_{b_{1} c_{1}}\right|$. A comparison against the relative variance of the mobility (in blue) highlights not only the non-linear mapping between the two uncertainties, but more importantly, the overall increase in variance following the matrix inversion. This is consistent with the often expressed view that inversion amplifies experimental errors. Once again, the peak condition number does not coincide with regions of highest uncertainty in the impedance. This further poses the question as to whether the condition number is an appropriate indicator of the uncertainty associated with experimental inverse methods.

The condition number describes, roughly, the rate of change due to a matrix transformation and is independent of experimental uncertainty, i.e. it is a property of the physical system. As an example, consider the analytical mobility matrix of an arbitrary system. Free from any experimental uncertainty this mobility matrix will have a large condition number at resonance, where the dynamics are dominated by a single mode. The condition number is often described as the maximum bound (worst case) on the magnification of the relative error on the input that is propagated onto the solution. With the above in mind, given a uniform operational velocity with uniformly distributed 
uncertainty, the condition number might be expected to correctly indicate regions of greater uncertainty. However, such an operational velocity is unlikely to occur in reality. In fact, it may be argued that in regions of high condition number, i.e. at resonance, the measured responses are less likely to be effected by noise owing to their increased SNR. Similarly, at resonance, the measured mobilities are inherently stable and therefore less likely to be affected by operator uncertainty. It is arguable therefore that the proposed inverse uncertainty propagation framework may be applied more generally to indicate regions of greater experimental uncertainty.

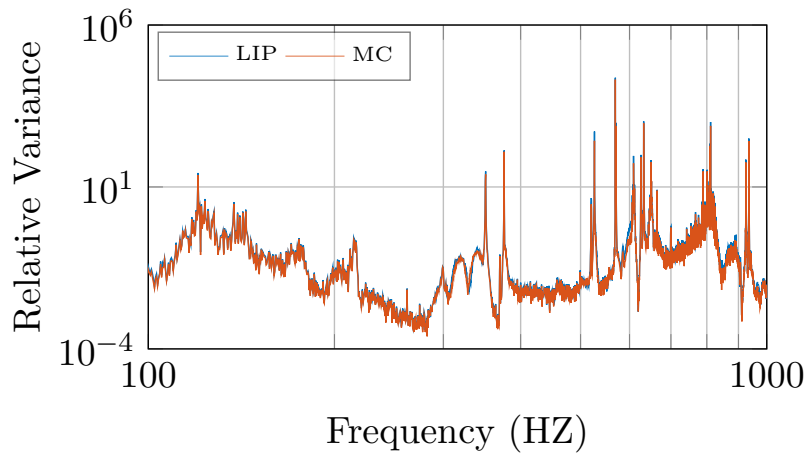

(a) Relative variance in the real part of the impedance, $\operatorname{Rel} \operatorname{Var}\left\{\mathfrak{R}\left(Z_{11}\right)\right\}$.

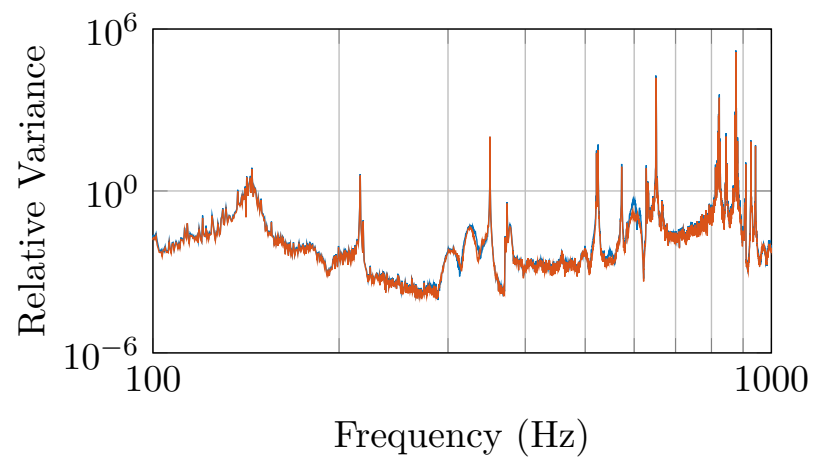

(b) Relative variance in the imaginary part of the impedance, $\operatorname{RelVar}\left\{\mathfrak{J}\left(Z_{11}\right)\right\}$.

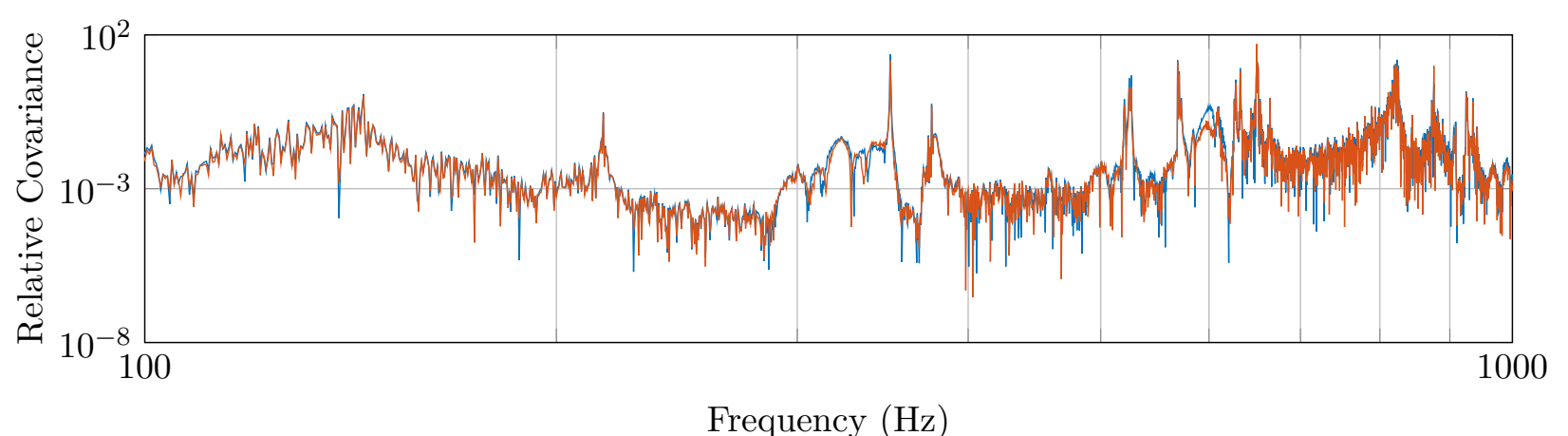

(c) Relative covariance between the real and imaginary parts of the impedance, $\operatorname{RelCov}\left\{\mathfrak{R}\left(Z_{11}\right), \mathfrak{J}\left(Z_{11}\right)\right\}$.

Figure 12: A comparison of the estimated impedance uncertainty based on the Linear-Inverse-Propagation (LIP) and a Monte-Carlo (MC) simulation.

Following the estimation of the impedance uncertainty the blocked force covariance matrix, $\Sigma_{\mathbf{f}} \in \mathbb{R}^{4 \times 4}$, is calculated as per equation 23. Shown in figure 13 are the predicted blocked force magnitudes alongside their $95 \%$ confidence intervals, assuming a log-normal distribution. Two sets of confidence intervals are shown. These are associated with the active and passive uncertainties. It can be seen that for the levels of uncertainty considered the active uncertainty contributes largely at higher frequencies, whilst the passive uncertainty is dominant at low frequencies.

Having calculated the blocked force, we are now able to predict an operational response at the remote reference DoF, $\tilde{b}$, i.e. perform an on-board validation. To acquire an estimate of the uncertainty in this prediction, the blocked force uncertainty was propagated through the forward model, as per equation 49. Shown in figure 14 is the result of the on-board validation alongside the $95 \%$ confidence intervals associated with the blocked force and forward mobility. The results clearly illustrate regions of increased uncertainty and that these largely coincide with disagreement in the predicted response, particularly around $160 \mathrm{~Hz}, 320 \mathrm{~Hz}$ and $600 \mathrm{~Hz}$. Additionally, is interesting to note that the blocked force is not necessarily the dominant source of uncertainty, and that the uncertainty of the mobility used in the forward calculation also contributes significantly, in other words, inversion is not always the dominant cause of uncertainty in the solution. 


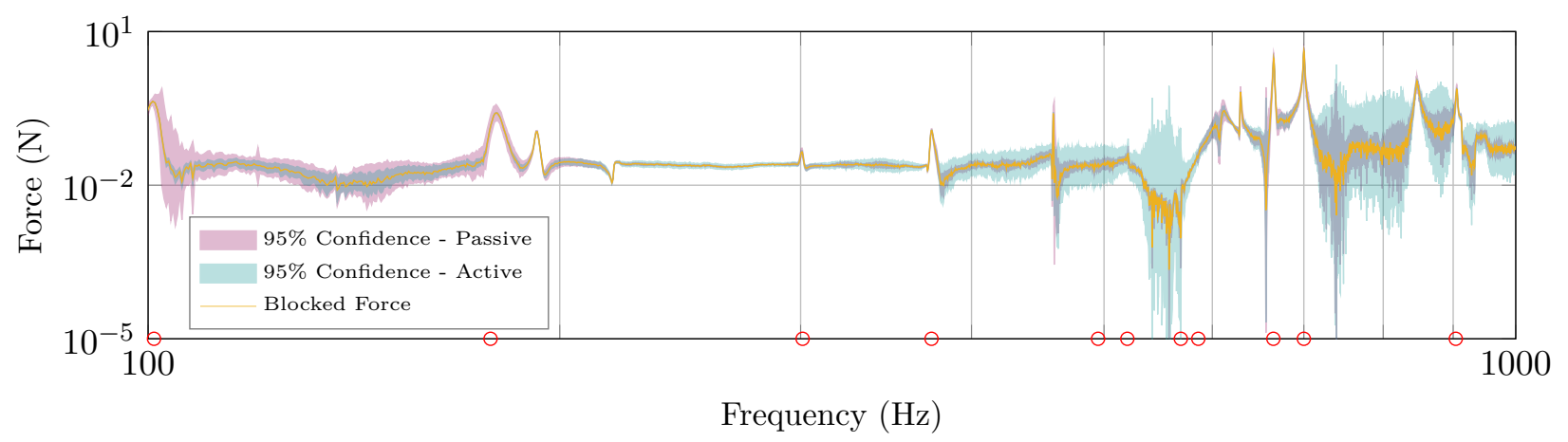

(a) Magntiude of the predicted blocked force, $\left|\bar{f}_{c_{1}}\right|$.

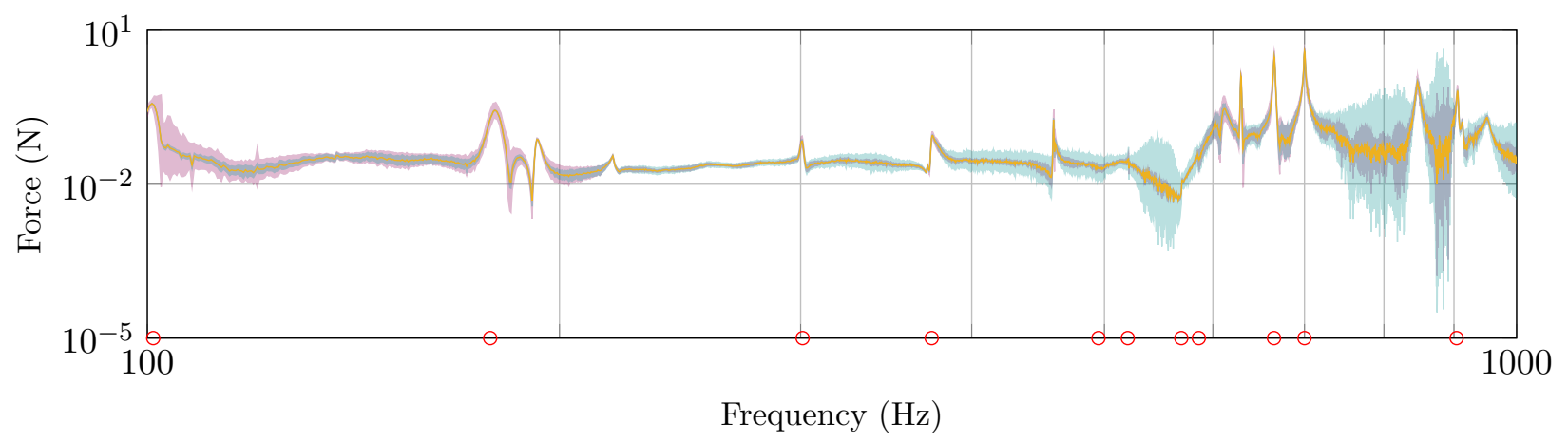

(b) Magntiude of the predicted blocked force, $\left|\bar{f}_{c_{2}}\right|$.

Figure 13: Inversely determined blocked forces at interface DoFs $c_{1}$ and $c_{2}$. Also shown are the 95\% confidence intervals associated with the active (velocity) and passive (mobility) uncertainty.

Whilst the estimated $95 \%$ bounds in figure 14 encapsulate much of the measured response, there are regions in which the bounds are exceeded, i.e. around $180 \mathrm{~Hz}, 550 \mathrm{~Hz}$, and $610 \mathrm{~Hz}$. These discrepancies do not appear to be due to the experimental or source based uncertainties and are likely a result of model uncertainty. Although resilient elements were used to mitigate the severity of model uncertainty, its effects are largely unavoidable. Nevertheless the estimated uncertainties appear sensible given the limitations of the proposed framework.

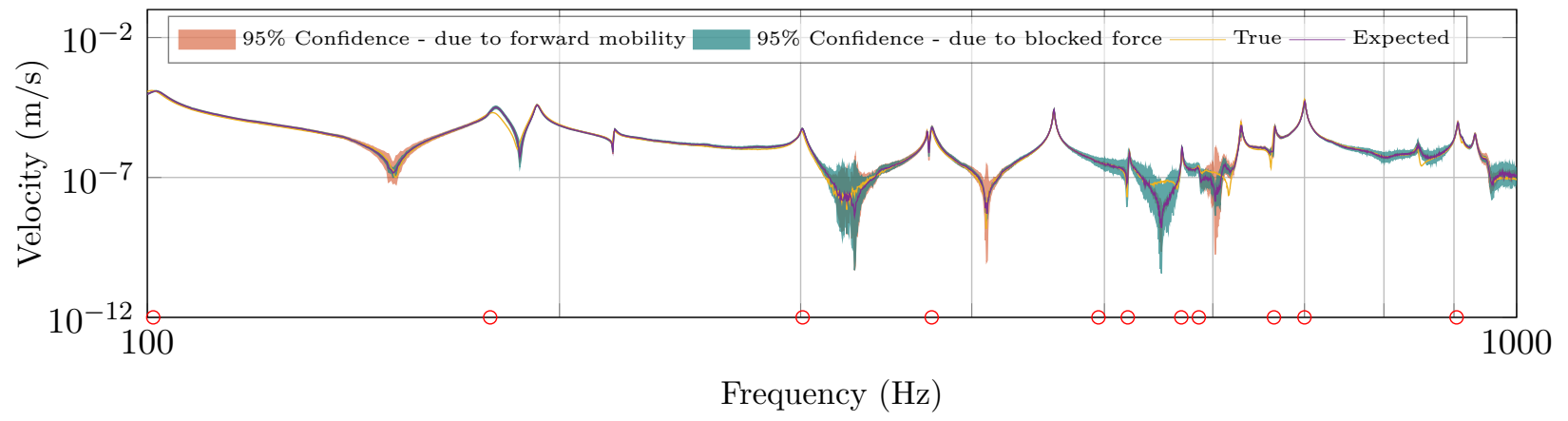

Figure 14: On-board validation result - Predicted and directly measured remote receiver velocity, $v_{\tilde{b}}$. Also shown, separately, are the $95 \%$ confidence bounds associated with the blocked force and forward mobility separately.

Although relatively simplistic, the above case study incorporates dynamic behaviour typical of more complex systems and illustrates the application of the proposed inverse propagation framework in establishing the uncertainties of inversely determined blocked forces, and those associated with predicted quantities. 


\section{Conclusions}

This paper was concerned with the development of a theoretical framework for the propagation of source and experimental uncertainty through a (complex and potentially over-determined) inverse procedure so as to yield an estimate of the uncertainties associated with in-directly determined blocked forces. A covariance based formulation was presented based on the general law of error propagation and the active/passive categorisation of uncertainty.

An essential step in the application of the proposed framework involves the propagation of measured FRF uncertainty through a matrix inversion. Two procedures were discussed: the first, a Linear-Inverse-Propagation (LIP); the second, a Monte-Carlo (MC) inversion. The LIP formulae derived may be considered a generalisation of those within the physics literature for complex non-square matrices. For small levels of uncertainty, the LIP and MC procedures were shown to be in good agreement.

The framework was further demonstrated, and validated, through two example studies: the first a numerical force reconstruction, and the second an experimental blocked force on-board validation. The framework was shown to provide uncertainties in agreement with MC predictions, which when propagated through a forward prediction, were largely in agreement with the discrepancies between measured and predicted quantities.

As a final remark, although it is often expressed that inversion errors are associated with ill conditioning, this was not found to be the case in either of the two case studies. Hence, it was argued that condition number may not be an appropriate indicator of uncertainty in vibro-acoustic inverse problems, and that a more rigorous treatment be considered.

\section{Acknowledgements}

This work was funded through the EPSRC Research Grant EP/P005489/1 Design by Science. The authors would like to acknowledge A.Clot and R.S.Langley at the University of Cambridge for their involvement in this work.

\section{Appendix A. Law of Error Propagation}

In this section we will derive, for completeness, the law of error propagation. We begin by considering the variables $x_{i}$ and $x_{j}$, generally, as the outputs of some multi-variable function,

$$
\begin{gathered}
x_{i}=G_{i}\left(a_{1}, a_{2}, a_{3}, \cdots\right)=G_{i}(\mathbf{a}) \\
x_{j}=G_{j}\left(a_{1}, a_{2}, a_{3}, \cdots\right)=G_{j}(\mathbf{a}) .
\end{gathered}
$$

Suppose that we repeatedly measure values of the input variables a, denoting the $n$th measurement by the subscript $n$. It is clear that a small change in the value of a will lead to a small change in the value of the output variables $x_{i}$ and $x_{j}$. Assuming that these changes remain small, they may be approximated to first order by a Taylor series expansion.

$$
\begin{gathered}
\Delta x_{i_{n}}=\frac{\partial G_{i}(\mathbf{a})}{\partial a_{1}} \Delta a_{1_{n}}+\frac{\partial G_{i}(\mathbf{a})}{\partial a_{2}} \Delta a_{2_{n}}+\cdots+\frac{\partial G_{i}(\mathbf{a})}{\partial a_{l}} \Delta a_{l_{n}} \\
\Delta x_{j_{n}}=\frac{\partial G_{j}(\mathbf{a})}{\partial a_{1}} \Delta a_{1_{n}}+\frac{\partial G_{j}(\mathbf{a})}{\partial a_{2}} \Delta a_{2_{n}}+\cdots+\frac{\partial G_{j}(\mathbf{a})}{\partial a_{m}} \Delta a_{m_{n}}
\end{gathered}
$$

We are interested in determining the covariance between the output variables $x_{i}$ and $x_{j}$. As such, we begin by multiplying together equation A.3 and A.4,

$$
\Delta x_{i_{n}} \Delta x_{j_{n}}=\left(\sum_{l} \frac{\partial G_{i}(\mathbf{a})}{\partial a_{l}} \Delta a_{l_{n}}\right)\left(\sum_{m} \frac{\partial G_{j}(\mathbf{a})}{\partial a_{m}} \Delta a_{m_{n}}\right) .
$$


Expanding the brackets,

$$
\Delta x_{i_{n}} \Delta x_{j_{n}}=\frac{\partial G_{i}(\mathbf{a})}{\partial a_{1}} \Delta a_{1_{n}}\left(\sum_{m} \frac{\partial G_{j}(\mathbf{a})}{\partial a_{m}} \Delta a_{m_{n}}\right)+\frac{\partial G_{i}(\mathbf{a})}{\partial a_{2}} \Delta a_{2_{n}}\left(\sum_{m} \frac{\partial G_{j}(\mathbf{a})}{\partial a_{m}} \Delta a_{m_{n}}\right)+\cdots+\frac{\partial G_{i}(\mathbf{a})}{\partial a_{l}} \Delta a_{l_{n}}\left(\sum_{m} \frac{\partial G_{j}(\mathbf{a})}{\partial a_{m}} \Delta a_{m_{n}}\right)
$$

and summing over $N$ measurements whilst dividing both sides by $\frac{1}{N-1}$, and noting the covariance relation $\sigma_{a_{l} a_{m}}=$ $\frac{1}{N-1} \sum_{n}^{N} \Delta a_{l_{n}} \Delta a_{m_{n}}$

$$
\begin{aligned}
\sigma_{x_{i} x_{j}}=\left(\frac{\partial G_{i}(\mathbf{a})}{\partial a_{1}} \frac{\partial G_{j}(\mathbf{a})}{\partial a_{1}} \sigma_{a_{1} a_{1}}+\frac{\partial G_{i}(\mathbf{a})}{\partial a_{1}} \frac{\partial G_{j}(\mathbf{a})}{\partial a_{2}} \sigma_{a_{1} a_{2}}+\cdots+\frac{\partial G_{i}(\mathbf{a})}{\partial a_{1}} \frac{\partial G_{j}(\mathbf{a})}{\partial a_{m}} \sigma_{a_{1} a_{m}}\right) \\
+\left(\frac{\partial G_{i}(\mathbf{a})}{\partial a_{2}} \frac{\partial G_{j}(\mathbf{a})}{\partial a_{1}} \sigma_{a_{2} a_{1}}+\frac{\partial G_{i}(\mathbf{a})}{\partial a_{2}} \frac{\partial G_{j}(\mathbf{a})}{\partial a_{2}} \sigma_{a_{2} a_{2}}+\frac{\partial G_{i}(\mathbf{a})}{\partial a_{2}} \frac{\partial G_{j}(\mathbf{a})}{\partial a_{m}} \sigma_{a_{2} a_{m}}+\cdots\right)+\cdots \\
\cdots+\left(\frac{\partial G_{i}(\mathbf{a})}{\partial a_{l}} \frac{\partial G_{j}(\mathbf{a})}{\partial a_{1}} \sigma_{a_{l} a_{1}}+\frac{\partial G_{i}(\mathbf{a})}{\partial a_{l}} \frac{\partial G_{j}(\mathbf{a})}{\partial a_{2}} \sigma_{a_{l} a_{2}}+\cdots+\frac{\partial G_{i}(\mathbf{a})}{\partial a_{l}} \frac{\partial f(\mathbf{a})}{\partial a_{m}} \sigma_{a_{l} a_{m}}\right) .
\end{aligned}
$$

The above may be written more conveniently in summation notation as,

$$
\sigma_{x_{i} x_{j}}=\sum_{l}^{M} \frac{\partial G_{i}(\mathbf{a})}{\partial a_{l}} \frac{\partial G_{j}(\mathbf{a})}{\partial a_{l}} \sigma_{a_{l} a_{l}}+\sum_{m \neq l} \frac{\partial G_{i}(\mathbf{a})}{\partial a_{l}} \frac{\partial G_{j}(\mathbf{a})}{\partial a_{m}} \sigma_{a_{l} a_{m}}
$$

or in matrix form as,

$$
\sigma_{x_{i} x_{j}}=\mathbf{J}_{\mathbf{i}} \boldsymbol{\Sigma}_{\mathbf{a}} \mathbf{J}_{\mathbf{j}}^{\mathbf{T}}
$$

where,

and

$$
\mathbf{J}_{\mathbf{i}}=\left[\begin{array}{llll}
\frac{\partial G_{i}(\mathbf{a})}{\partial a_{1}} & \frac{\partial G_{i}(\mathbf{a})}{\partial a_{2}} & \cdots & \frac{\partial G_{i}(\mathbf{a})}{\partial a_{M}}
\end{array}\right]
$$

$$
\mathbf{J}_{\mathbf{j}}=\left[\begin{array}{llll}
\frac{\partial G_{j}(\mathbf{a})}{\partial a_{1}} & \frac{\partial G_{j}(\mathbf{a})}{\partial a_{2}} & \ldots & \frac{\partial G_{j}(\mathbf{a})}{\partial a_{M}}
\end{array}\right]
$$

are the Jacobians of the functions $x_{i}=G_{i}(\mathbf{a})$ and $x_{j}=G_{j}(\mathbf{a})$, respectively, and $\boldsymbol{\Sigma}_{\mathbf{a}}$ is the covariance matrix between the elements of the input vector $\mathbf{a}$,

$$
\boldsymbol{\Sigma}_{\mathbf{a}}=\left[\begin{array}{cccc}
\sigma_{a_{1} a_{1}} & \sigma_{a_{1} a_{2}} & \cdots & \sigma_{a_{1} a_{M}} \\
\sigma_{a_{2} a_{1}} & \sigma_{a_{2} a_{2}} & \cdots & \sigma_{a_{2} a_{M}} \\
\vdots & \vdots & \ddots & \vdots \\
\sigma_{a_{M} a_{1}} & \sigma_{a_{M} a_{2}} & \cdots & \sigma_{a_{M} a_{M}}
\end{array}\right] .
$$

Equations A.9-A.12 describe, generally, the law of error propagation.

\section{Appendix B. Free-Free Beam Model}

The translational mobility of a free-free beam excited at an arbitrary point, $x_{j}$, for an arbitrary receiver position, $x_{i}$, is given by [54] as,

$$
\begin{array}{rr}
Y_{i j}=\frac{i \omega}{2 \bar{B} k^{3}}\left(f_{1}\left(x_{j}\right) g_{1}\left(x_{i}\right)+f_{2}\left(x_{j}\right) g_{2}\left(x_{i}\right)\right) & 0 \leq x_{i} \leq x_{j} \\
Y_{i j}=\frac{i \omega}{2 \bar{B} k^{3}}\left(f_{1}\left(x_{i}\right) g_{1}\left(x_{j}\right)+f_{2}\left(x_{i}\right) g_{2}\left(x_{j}\right)\right) & x_{j} \leq x_{i} \leq l
\end{array}
$$

where, $i=\sqrt{-1}$ is the imaginary unit, $\bar{B}$ is the beam bending stiffness, $k$ is the bending wave number, $l$ is the beam length and;

$$
f_{1}(x)=\cosh k x-\cosh k(l-x) \cos k l-\sinh k(l-x) \sin k l-\cos k x+\cos k(l-x) \cosh k l-\sinh k l \sin k(l-x)
$$




$$
\begin{gathered}
f_{2}(x)=\sinh k x-\sinh k(l-x) \cos k l-\sin k l \cosh k(l-x)-\sin k x+\sinh k l \cos k(l-x)-\cosh k l \sin k(l-x) \\
g_{1}(x)=-\frac{(\sin k x+\sinh k x)}{2(1-\cosh k l \cos k l)} \\
g_{2}(x)=\frac{(\cos k x+\cosh k x)}{2(1-\cosh k l \cos k l)} .
\end{gathered}
$$

The angular velocity and moment mobilities are obtained by differentiating equations B.1-B.2 with respect to $x_{i}$ and $x_{j}$, respectively.

\section{References}

[1] T.T. Wolde and G.R. Gadefelt. Development of standard measurement methods for structureborne sound emission. Noise Control Engineering Journal, 28(1), 1987.

[2] F. Fahy and J. Walker. Advance Applications in Acoustics, Noise and Vibration. Spoon Press, 2004.

[3] A.T. Moorhouse, A.S. Elliott, and T.A. Evans. In situ measurement of the blocked force of structure-borne sound sources. Journal of Sound and Vibration, 325(4-5):679-685, sep 2009.

[4] M.V. van der Seijs, D. de Klerk, and D.J. Rixen. General framework for transfer path analysis: History, theory and classification of techniques. Mechanical Systems and Signal Processing, pages 1-28, 2015.

[5] D. de Klerk and D.J. Rixen. Component transfer path analysis method with compensation for test bench dynamics. Mechanical Systems and Signal Processing, 24(6):1693-1710, 2010.

[6] A.S. Elliott, A.T. Moorhouse, T. Huntley, and S. Tate. In-situ source path contribution analysis of structure borne road noise. Journal of Sound and Vibration, 332(24):6276-6295, 2013.

[7] D. Lennström, M. Olsson, F. Wullens, and A. Nykänen. Validation of the blocked force method for various boundary conditions for automotive source characterization. Applied Acoustics, 102:108-119, 2016.

[8] T. Alber, M. Sturm, and A.T. Moorhouse. Independent characterization of structure-borne sound sources using the in-situ blocked force method. In Proceedings of Internoise, pages 7302-7313, 2016.

[9] M. Sturm, T. Alber, A.T. Moorhouse, D. Zabel, and Z. Wang. The in-situ blocked force method for characterization of complex automotive structure-borne sound sources and its use for virtual acoustic prototyping. Isma, (October), 2016.

[10] M. Sturm, M. Yankonis, R. Bosch, C. Marchand, S. Sherman, J. Hirscher, M. Priebe, P. Parikh, and A.T. Moorhouse. Robust NVH Development of Steering Systems Using In-Situ Blocked Forces from Measurements with Low-Noise Driver Simulators. In Noise-Con, Grand Rapids, MI, 2017.

[11] D. Lennström. In-Situ Characterization of Vibrations from a Door Mounted Loudspeaker. SAE Technical Paper, 01:1-6, 2018.

[12] G. Banwell and R. Faventi. Assessment of experimental techniques to characterise the vibration source strength of a motor radially mounted with resilient elements. In ISMA, Leuven, 2016.

[13] A.S. Elliott and A.T. Moorhouse. In-situ characterisation of structure borne noise from a building mounted wind turbine. ISMA, pages 2055-2068, 2010.

[14] Joint Committee For Guides In Metrology. Evaluation of measurement data - Guide to the expression of uncertainty in measurement, 2008.

[15] C. Soize. A comprehensive overview of a non-parametric probabilistic approach of model uncertainties for predictive models in structural dynamics. Journal of Sound and Vibration, 288(3):623-652, 2005.

[16] C. Soize. Random matrix theory for modeling uncertainties in computational mechanics. Computer Methods in Applied Mechanics and Engineering, 194(12-16):1333-1366, 2005.

[17] Sondipon Adhikari. Wishart Random Matrices in Probabilistic Structural Mechanics. Journal of Engineering Mechanics, 134(12):1029-1044, 2008.

[18] S. Adhikari, L. Pastur, A. Lytova, and J. Du Bois. Eigenvalue density of linear stochastic dynamical systems: A random matrix approach. Journal of Sound and Vibration, 331(5):1042-1058, 2012.

[19] A. Acri, E. Nijman, A. Acri, and G. Offner. Influences of system uncertainties on the numerical transfer path analysis of engine systems. Mechanical Systems and Signal Processing, 95:106-121, 2017.

[20] J.S. Bendat. Statistical errors in measurement of coherence functions and input/output quantities. Journal of Sound and Vibration, 59(3):405421, 1978.

[21] J.S. Bendat and A.G. Piersol. Random Data: Analysis and Measurement Procedures. 2011.

[22] J.S. Bendat and A.G. Piersol. Engineering Applications of Correlation and Spectral Analysis. John Wiley \& Sons, Inc., 2 nd edition, 1993.

[23] G.H. James, D.C. Zimmerman, and R.L. Mayes. An experimental study of frequency response function (frf) based damage assessment tools. In Society for Experimental Mechanics, Inc, 16 th International Modal Analysis Conference, volume 1, pages 151-157, 1998.

[24] K. Worden. Confidence Bounds for Frequency Response Functions From Time Series Models. Mechanical Systems and Signal Processing, 12(4):559-569, 1998.

[25] B. Cauberghe, P. Guillaume, B. Dierckx, and P. Verboven. Identification of modal parameters from inconsistent data. (January 2002):809-815, 2014.

[26] D. de Klerk. Dynamic response characterization of complex systems through operational identification and dynamic substructuring. PhD thesis, TU Delft, 2009.

[27] S.N. Voormeeren, D. de Klerk, and D.J. Rixen. Uncertainty quantification in experimental frequency based substructuring. Mechanical Systems and Signal Processing, 24(1):106-118, 2010. 
[28] D.J. Rixen. How measurement inaccuracies induce spurious peaks in frequency based substructuring. Proceedings of the Twenty Sixth International Modal Analysis Conference, Orlando, FL. Society for Experimental Mechanics, Bethel, CT, 2008.

[29] P. Gajdatsy, P. Sas, W. Desmet, K. Janssens, and H. van der Auweraer. Effect of systematic FRF errors on matrix inversion based vibro-acoustic analysis methods. In Sensors, Instrumentation and Special Topics, Conference Proceedings of the Society for Experimental Mechanics Series, volume 6, pages 197-206, 2011.

[30] D. de Klerk and R. Visser. Characterization of Measurement Errors in Experimental Frequency Based Substructuring. ISMA 2010 including USD2010, pages 1881-1890, 2010.

[31] D. de Klerk. How Bias Errors Affect Experimental Dynamic Substructuring. 3:1101-1112, 2011.

[32] J.W.R. Meggitt. On the treatment of uncertainty in experimentally measured frequency response functions (Submitted). Metrologia, 2018.

[33] N.M. Ridler and M.J. Slater. An approach to the treatment of uncertainty in complex -parameter measurements. Metrologia, 39(3):295-302, 2002.

[34] B. D. Hall. On the propagation of uncertainty in complex-valued quantities. Metrologia, 41(3):173-177, 2004.

[35] T. Schultz, M. Sheplak, and L.N. Cattafesta. Application of multivariate uncertainty analysis to frequency response function estimates. Journal of Sound and Vibration, 305(1-2):116-133, 2007.

[36] H.S. Kim and T.L. Schmitz. Bivariate uncertainty analysis for impact testing. Measurement Science and Technology, 18(11):3565-3571, 2007.

[37] A.N. Thite and D.J. Thompson. The quantification of structure-borne transmission paths by inverse methods. Part 2: Use of regularization techniques. Journal of Sound and Vibration, 264(2):433-451, 2003.

[38] A.N. Thite and D.J. Thompson. The quantification of structure-borne transmission paths by inverse methods. Part 1: Improved singular value rejection methods. Journal of Sound and Vibration, 264(2):411-431, 2003.

[39] S.E.S. Karlsson. Identification of External Structural Loads From Measured Harmonic Responses. Journal of Sound and Vibration, 196(1):59-74, 1996.

[40] M.W.F. Wernsen, M.V. van der Seijs, and D. de Klerk. An indicator sensor criterion for in-situ characterisation of source vibrations. In Conference Proceedings of the Society for Experimental Mechanics, number 5. Springer, 2017.

[41] M. Blau. Indirect Measurement of Multiple Excitation Force Spectra by FRF Matrix Inversion : Influence of Errors in Statistical Estimates of FRFs and Response Spectra. Acustica, 85(1):464-479, 1999.

[42] J.R. Fonseca, M.I. Friswell, J.E. Mottershead, and A.W. Lees. Uncertainty identification by the maximum likelihood method. Journal of Sound and Vibration, 288(3):587-599, 2005.

[43] E. Zhang, J. Antoni, and P. Feissel. Bayesian force reconstruction with an uncertain model. Journal of Sound and Vibration, 331(4):798-814, 2012.

[44] Armen Der Kiureghian and Ove Ditlevsen. Aleatory or epistemic? Does it matter? Structural Safety, 31(2):105-112, 2009.

[45] R. Penrose and J.A. Todd. A generalized inverse for matrices. Mathematical Proceedings of the Cambridge Philosophical Society, 51(03):406, 1955 .

[46] A.S. Elliott, J.W.R. Meggitt, and A.T. Moorhouse. Blocked forces for the characterisation of structure borne noise. In Internoise2015, pages 5798-5805, San Fransisco, 2015.

[47] J.W.R. Meggitt, A.T. Moorhouse, and A.S. Elliott. On the problem of describing the coupling interface between sub-structures : an experimental test for 'completeness'. In IMAC XVIII, pages 1-11, Orlando, 2018.

[48] S. Mustonen. A measure for total variability in multivariate normal distribution. Computational Statistics $\mathcal{E}$ Data Analysis, 23(3):321-334, 1997.

[49] K. Ackerstaff and Et al. Measurement of the one-prong hadronic tau branching ratios at LEP. Eur.Phys.J.C4, pages 193-206, 1998.

[50] M. Lefebvre, R. K. Keeler, R. Sobie, and J. White. Propagation of errors for matrix inversion. Nuclear Instruments and Methods in Physics Research, Section A: Accelerators, Spectrometers, Detectors and Associated Equipment, 451(2):520-528, 2000.

[51] A. Hjorunges. Complex-Valued Matrix Derivatives. Cambridge University Press, 1 edition, 2011.

[52] J.W.R. Meggitt, A.S. Elliott, A.T. Moorhouse, G. Banwell, H. Hopper, and J. Lamb. Broadband characterisation of in-duct acoustic sources using an equivalent source approach (Submitted). Journal of Sound and Vibration, 2018.

[53] V. Cotoni, R.S. Langley, and M.R.F. Kidner. Numerical and experimental validation of variance prediction in the statistical energy analysis of built-up systems. Journal of Sound and Vibration, 288(3):701-728, 2005.

[54] J.X. Su. Simplified characterisation of structure-borne sound sources with multi-point connections. Phd, University of Liverpool, 2003. 